\title{
An Accurate Analytical Model for 802.11e EDCA under Different Traffic Conditions with Contention-Free Bursting
}

\author{
Nada Chendeb Taher, ${ }^{1,2,3}$ Yacine Ghamri-Doudane, ${ }^{1}$ \\ Bachar El Hassan, ${ }^{3}$ and Nazim Agoulmine ${ }^{2}$ \\ ${ }^{1}$ ENSIIE, 1 Square de la Résistance, 91025 Evry Cedex, France \\ ${ }^{2}$ University of Evry val d'Essonne, Boulevard François Mitterrand, 91025 Evry Cedex, France \\ ${ }^{3}$ Faculty of Engineering, Lebanese University, El Arz street, El Kobbeh, Tripoli, Lebanon
}

Correspondence should be addressed to Nada Chendeb Taher, chendeb@ensiie.fr

Received 25 September 2010; Revised 24 January 2011; Accepted 2 March 2011

Academic Editor: Abdallah Shami

Copyright (C) 2011 Nada Chendeb Taher et al. This is an open access article distributed under the Creative Commons Attribution License, which permits unrestricted use, distribution, and reproduction in any medium, provided the original work is properly cited.

\begin{abstract}
Extensive research addressing IEEE 802.11e enhanced distributed channel access (EDCA) performance analysis, by means of analytical models, exist in the literature. Unfortunately, the currently proposed models, even though numerous, do not reach this accuracy due to the great number of simplifications that have been done. Particularly, none of these models considers the 802.11e contention free burst (CFB) mode which allows a given station to transmit a burst of frames without contention during a given transmission opportunity limit (TXOPLimit) time interval. Despite its influence on the global performance, TXOPLimit is ignored in almost all existing models. To fill in this gap, we develop in this paper a new and complete analytical model that (i) reflects the correct functioning of EDCA, (ii) includes all the 802.11e EDCA differentiation parameters, (iii) takes into account all the features of the protocol, and (iv) can be applied to all network conditions, going from nonsaturation to saturation conditions. Additionally, this model is developed in order to be used in admission control procedure, so it was designed to have a low complexity and an acceptable response time. The proposed model is validated by means of both calculations and extensive simulations.
\end{abstract}

\section{Introduction}

In recent years, IEEE 802.11 wireless LANs has gradually become the preferred technology for wireless Internet and Intranet access. However, there are many inherent QoS limitations in the base standard, as it was basically developed to serve best effort data services. With the increasing demand for real-time multimedia application support, a new standard amendment, IEEE 802.11e, has been specified [1]. It aims to support QoS by providing differentiated classes of service in the medium access control (MAC) layer so that it can deliver time-critical multimedia traffic in addition to traditional data packets. However, despite these enhancements of the MAC layer, this new standard still cannot guarantee the required QoS for real-time multimedia applications such as voice and video. For this reason, and as it stands, the IEEE 802.11e WLAN standard cannot be used as a framework for multimedia applications. In fact, when the network is not fully saturated, the service differentiation works properly and gives the multimedia traffic the opportunity to be served with a good QoS. However, a problem arises when the network starts to reach the saturation state. In this case, all traffics suffer from high collision rate, and the QoS for multimedia applications is degraded.

To solve this problem and to extend the capability of 802.11e WLAN to deliver multimedia traffic with success, an efficient admission control must be used jointly with the 802.11e standard. Hence, an efficient admission control algorithm will take a decision based on the performance measurements at each new traffic arrival. To be able to guarantee the QoS requirements in terms of required bandwidth and tolerated delay, this algorithm must have as input parameters, the accurate values of the achievable throughput and access delay. Therefore, it is necessary to develop an analytical model of the protocol capable of delivering sufficiently accurate QoS metrics. 
Actually, in the recent years several analytical models for 802.11e EDCA had been proposed, each one has its own add-ins and enhancements by comparison to the previous ones, but each of these have also its lacks and limitations. All these models aim to deliver an estimation of the per-AC throughput and/or access delay. Several questions are addressed in this paper. (i) Do these models allow modeling finely the EDCA behavior and can they correctly reflect its functioning and, therefore, accurately predict its performance? (ii) Are these models complete and can they be applicable to all situations and configurations? (iii) Which model best estimates the performance metrics so that it can be used as a basis of an admission control algorithm in the future?

Unfortunately, all the major existing analytical models are not sufficiently complete and therefore cannot correctly reflect the exact behavior of the protocol in all functioning conditions. This lack can be explained by the three following facts.

The Models Miss One or More Features of the Protocol. In fact, the addition of any of the missed features of the protocol into a given analytical model leads to a better accuracy and completeness. To understand this point, let us take a simple example. The analytical model proposed by Bianchi [2] has been used and corrected by the addition of the retry limit feature into the Markov chain [3]. The authors of [3] showed in their evaluation study that this simple addition considerably enhances the analytical model and gives more accurate results. Other illustrative examples will be later detailed in the paper in order to highlight this important accuracy enhancement when the model is improved by the inclusion of a baseline feature.

The Models Either Show a Misunderstanding of the Standard Specification or Use an Old Version. Some analytical models have been developed based on the old specification of the 802.11e EDCA, named Enhanced distributed coordination function (EDCF) [4-6], and some others, though based on the final standard [1], did not model accurately some features of the protocol, especially the arbitration interframe space (AIFS) countdown procedure and its cooperation with the backoff procedure [7-9]. These models do not correctly reflect the behavior of the protocol resulting in a degradation of their accuracy.

The Models Do Not Take into Account the Three Differentiation Parameters. Almost all of the major analytical models do not consider the TXOPLimit which is an important differentiation parameter. The TXOPLimit is one of the major contributions to the 802.11 e standard; it clearly has a major role in applications that need a large bandwidth such as video [10]. This will be shown again later in this paper. Despite its importance, TXOPLimit is ignored in almost all existing models. So, a model without TXOPLimit can only be used if this parameter is turned down, which makes it incomplete and unable to be applied to all configurations and situations.
For these three main reasons, we found out that there is a compelling need for a new, more accurate and complete analytical model. Such model is the mandatory first step towards an efficient admission control algorithm. To achieve the required level of correctness, accuracy and completeness, we proceed through several steps that describe our main contributions in this paper.

(i) First of all, we gained an in depth understanding of IEEE 802.11e EDCA as defined by the final standard [1]. This clear understanding allowed us to identify the different steps through which an AC can transit during its transmission cycle and, therefore, to integrate all the features of the protocol in our model and to correctly model the interactions among features as well (e.g., AIFS and backoff countdown procedures).

(ii) Being the major missing part in all existing models, the effect of TXOPLimit differentiation parameter on the global performance is studied thoroughly in this paper. A solution is proposed as well to integrate it into the complete analytical model.

(iii) We have developed a new and complete analytical model using a four-dimensional discrete time Markov chain in general network conditions. The resulting model can deliver, for each $\mathrm{AC}$, the achievable throughput and access delay by means of two close-form numerical equations. The accuracy of this model is evaluated and demonstrated through several usage scenarios.

These contributions are detailed in the different sections of this paper. After this introduction, a brief description of the main characteristics of the 802.11e EDCA MAC protocol and its operation will be reviewed. Section 3 contains a study of a large set of major models existing in the related literature. This study will show the add-ins and contributions of these models. It will also show us in parallel the lacks and the problems to be overcome. The effect of the TXOPLimit differentiation parameter on the global network performance and our approach to analytically model this parameter constitute the subject of Section 4 . To be able to compare our model to the most successful one, which assumes saturation conditions, we will start with a presentation of our analytical model in the saturation conditions. So in Section 5, we present the details of the way we proceed to cover all the features of the $802.11 \mathrm{e}$ EDCA, and to reflect its real functioning. The saturation model is validated by means of simulations and comparison with a representative already-existing one, in Section 6 . Having in mind that saturation assumption is a limitation for such models, we have extended the model to cover all network conditions, and we presented the resulting complete model in Section 7. Extensive simulations to validate the entire model are given, analyzed, and discussed in Section 8. To complete our work, the computational aspects related to the numerical resolution of our model are discussed in Section 9. Finally, our conclusions and perspectives are drawn in Section 10. 


\section{802.11e EDCA MAC Access Mechanism}

In IEEE 802.11e, a new MAC access method named hybrid coordination function (HCF) is introduced [1]. The HCF consists of two parts. One of them is the HCF contentionbased channel access mechanism; also named Enhanced distributed channel access (EDCA). The other one is the HCF controlled channel access (HCCA). EDCA is the fundamental and mandatory mechanism of IEEE 802.11e, while HCCA is optional and requires centralized polling and scheduling algorithms to allocate the resources. In this paper, our work covers only the mandatory EDCA access mechanism.

EDCA handles application needs by classifying their traffic into four Access Categories (ACs): AC_BK for background traffic, AC_BE for best effort traffic, AC_VI for the video traffic and $\mathrm{AC}_{-} \mathrm{VO}$ for voice. Each $\mathrm{AC}$ has its own priority access parameters. These parameters are neither constant nor fixed by the physical layer as with DCF (DCF is the distributed coordination function, the mandatory access mechanism of the base 802.11 standard). They are assigned by a QoS access point (QAP). These differentiation parameters are basically: the arbitration interframe space number (AIFSN), the contention window (CW) represented by its minimum and maximum limits $\left(\mathrm{CW}_{\min }, \mathrm{CW}_{\max }\right)$, and the transmission opportunity limit (TXOPLimit) [1]. So, three parameters are used in EDCA to implement AC specific traffic prioritization, that is, AIFS, CW and TXOPLimit. EDCA assigns smaller CW bounds, smaller AIFS and greater TXOPLimit to ACs with higher priorities and vice versa.

Let us now see how an AC operates under the EDCA basic access mechanism. In EDCA, each AC within a wireless station behaves like a virtual station; it must sense the medium before initiating a transmission. If the medium is sensed as being idle for a time interval equal to its specific AIFS $[\mathrm{AC}]$, then it starts a backoff process by choosing a random waiting time between 0 an $\mathrm{CW}$ timeslots, and then decrements the counter. At any time slot during the backoff process, if the AC senses the channel busy, it freezes its backoff counter and waits for the channel to be idle again for a complete AIFS [AC] before continuing the backoff countdown. Once the backoff counter reaches zero, the AC transmits into the channel. Acknowledgements (ACKs) are used to notify the sending station about the successful reception of a frame. If no acknowledgement is received, the sending station considers that there is a collision or channel error. In this case, it waits for an AIFS and schedules a retransmission. Other stations, which are not involved in the collision, wait for a specific extended inter frame space (EIFS $[\mathrm{AC}]$ ) which is greater than the AIFS [AC]. This is called the post-collision and is used to give the sending station the priority to retransmit as soon as possible. When a collision occurs among different ACs within the same station, the higher priority $\mathrm{AC}$ is granted the opportunity for physical transmission.

After each unsuccessful transmission, the $\mathrm{CW}$ is doubled until the $\mathrm{CW}_{\max }[\mathrm{AC}]$ is reached. The starting value of $\mathrm{CW}$ for a specific $\mathrm{AC}$ is $\mathrm{CW}_{\min }[\mathrm{AC}]$. The frame is dropped after a maximum number of retransmissions called retry limit.
After each successful transmission, the transmitting AC initiates another random backoff, even if there is no other pending packet to be delivered. This is often referred to as post-backoff, as this backoff is done after, not before a transmission. The post-backoff guarantees that there is always one random backoff time between two consecutive frame exchanges [1]. After achieving the post backoff procedure, if the station has data to be transmitted, a new channel access operation is initiated. Otherwise, the station stays in an idle state waiting for the arrival of new packets.

It is also important to note that IEEE 802.11e defines the TXOPLimit as the time interval during which a particular station has the right to transmit. During a TXOPLimit, a station may be allowed to transmit multiple data frames from the same AC with a short inter frame space (SIFS) between an acknowledgment (ACK) and the subsequent data frame [1]. This process is also referred to as contention free burst (CFB) or TXOP bursting. The duration of a TXOP is the time during which the TXOP holder maintains an uninterrupted control of the medium. A zero value for TXOPLimit indicates that a single frame may be transmitted for each TXOP.

In addition to the basic access mechanism described above, an optional four-way handshaking technique, known as request-to-send/clear-to-send (RTS/CTS) has been standardized. So, instead of transmitting a data frame, a short RTS is transmitted and the data frames wait for the reception of the CTS prior to its transmission. This technique permits to "reserve" the channel before transmission in order to reduce the collision rate especially when the data frames are greater than a specific value called RTSThreshold.

\section{Related Works and Motivations}

In the recent years, a paramount number of studies appeared in the literature investigating the performance of 802.11 DCF and 802.11e EDCA protocol. This performance has been explored by means of not only simulations but also by means of analytical models, with the goal being to either predict analytically performance metrics or to understand the protocol behavior.

Two types of analytical models exist in the literature, the first one is based on the discrete Markov chain model, and the second one is based on the mean value analysis. At the level of our knowledge, there is no accuracy comparison between these two approaches. But it is clear that the first approach gains a great success in the field of 802.11 modeling. The second one, despite its low computation complexity, omits system details and necessitates a high number of assumptions.

Alternatively, the analytical models proposed can be classified into two groups, the first one includes models under network saturation conditions and the second one is related to those applicable to all network conditions. The saturation assumption enables queuing dynamics to be neglected and avoids the need for detailed modeling of traffic characteristics. So, the saturation assumption greatly simplifies the modeling. On the other hand, although performance evaluation under saturation conditions provides the fundamental bounds on system throughput and delay, it 
cannot reveal the best working scenarios. It was proved by simulation, as well as by older models, that the maximum capacity of IEEE $802.11[10,11]$ and $802.11 \mathrm{e}[12,13]$ can only be achieved in the nonsaturated case. So, a framework capable of analyzing and predicting the performance under both saturation and non-saturation conditions can be very helpful in leading to first better understanding of the EDCA mechanism and, at a latter extent, facilitating the design of an accurate admission control algorithm.

Let us now show the value added and the limitations of the major already existing models for DCF and EDCA.

3.1. DCF Analytical Models. Earliest in this field, Bianchi [2] proposed a Markov chain to model the behavior of DCF in order to analyze the saturation throughput performance. $\mathrm{Wu}$ et al. [3] extends Bianchi's model to include finite packet retry limits. Ziouva and Antonakopoulos [14] introduces an additional transition state in order to confront the backoff suspension characteristic which is not present in [2]. This feature is also introduced in [15], but the authors consider that the channel-busy probability and the collision probability are the same, which is not a justified assumption. Indeed, these are two different probabilities as the former does not necessarily imply the latter. Vardakas et al. [16] uses Bianchi's model to calculate the end to end delay. In [17], the authors aim at reaching a sufficiently accurate approximation for the service time distribution while using the Bianchi's model too. For nonsaturated conditions, Malone et al. [10] extends [2] by adding post-backoff states, in which the station's queue is empty. Hung and Marsic [11] uses the same extension done in [10] to compute the effect of hidden stations. Finally, Tay and Chua [18] use the mean value analysis to get the saturation throughput.

This historical study of DCF analytical models shows two tendencies: some models use the Bianchi's model as it is to study other performance metrics such as the access delay, while some others concentrate on introducing missing features into the Bianchi's model and prove better accuracy. Indeed, the study of these different schemes showed that the integration of any missed feature into a given analytical model gives more accuracy and leads to more precise results.

3.2. EDCA Analytical Models. Several analytical models are also proposed for 802.11e EDCA; some of them concern the EDCF protocol defined by a former draft version of the standard, and the others use the standard version [19]. As we will show hereafter, almost all models extend the Bianchi's two-dimensional Markov chain model to take into account different AIFS and different CW but neglect the use of different TXOPLimit.

3.2.1. Under the Saturation Assumption. Xu et al. [4] propose an analytical model for EDCF, which is a multi dimensional Markov chain, where the dimension depends on the number of flows. Different aspects of the protocol are missing (retry limit and virtual collision as examples) and the calculation method is quite complex. Xiao [5] enlarges the original two-dimensional Markov chain to a three-dimensional one (even though some models are three dimensional Markov chains, the third dimension is constant. It allows only a good comprehension of the model. So, these models can be considered effectively as a two-dimensional one), and analyzes the effects of the different contention window sizes on the throughput However, the AIFS-based priority scheme is not included. Another lack in the model is that it considers that it is the collision probability that controls the backoff decrement and freeze; this is not exact as effectively, the backoff activity is controlled by the channel busy probability. So, the model proposed in [5] is simply a Bianchi's one with different CWs for each AC. In [6], Hui and Devetsikiotis borrow strengths from two different models, Bianchi [2] and Tay and Chua [18], and combine them to compose a new unified performance model for EDCA analysis as defined in a former draft version, without involving more complex Markov chain constructs. One should note, however, that the Markov chain used in [6] suffers from the same limitations as the Bianchi's one, that is, neither the retry limit feature, nor the backoff freeze characteristic of the EDCA are considered. Also, TXOP is neglected, and the AIFS final description and its cooperation with the backoff procedure are absent.

Robinson and Randhawa [20] study the effect of the post-collision period by the introduction of contention zonespecific transmission probability caused by the different AIFSs. The model brings something new in the field of EDCA modeling, but it is limited by the various approximations (e.g., Markov chain decomposition) made therein. Moreover, the model does not consider the virtual collision. It is limited to the case where each station has only one active AC. According to their proposed modeling, the extension of the model proposed to the general case where either several or all ACs are active in the same station is not a simple matter. In [7], Kong et al. focus on the possibility for a station's backoff procedure to be continuously suspended due to multiple consecutive transmissions from other stations. Contrarily to previous models, [7] covers an important number of EDCA features, but it still misses the TXOPLimit differentiation parameter and has an important lack in the AIFS modeling. Actually, the authors consider that during the AIFS waiting time, if the channel is detected to be busy by an AC, this latter freeze and defreeze again when the channel is sensed idle again. This is a miscomprehension of the EDCA procedure, as AIFS waiting cannot be frozen and has to restart from the beginning. The AIFS procedure is also a very important differentiation mechanism lacking in Xiao's model [21]. Xiao assumed equal AISFN to all ACs. So, Xiao's model only develops a two-dimensional Markov chain model for the case of general ACs.

Other models [22-24] have tried to handle the AIFS procedure by the use of three-dimensional Markov chain. However, since these models consider different ACs individually (a specific Markov chain for each AC, depending on special values of AIFS), the corresponding analysis procedure should also be separate, which makes the whole EDCA system modeling and analysis much more complicated. The proposed model cannot be applicable to the general case whatever the values of the differentiation parameters are. In [25], the authors extend the work of Tay and Chua [18] and 
use the mean value analysis to introduce the AIFS and the CW differentiation. Reference [25] also omits many system details and does not invoke the TXOP differentiation, it assumes a single AC active in a station, and analyzes only 2 ACs in the network. Furthermore, many features of EDCA are neglected. Virtual collisions and retry limit are examples. In [8], the authors reuse the concept of contention zone specific probabilities, similar to [20,21], without improving the accuracy of the AIFS differentiation. Also Xiong and Mao [26] combine the work of $[7,20]$, but their study is still limited to one AC per station and cannot be extended. Recently, Yan and Pan in [27] try to consider correctly the AIFS and backoff cooperation process in EDCA as defined in the standard [1], but unfortunately, their analysis includes some strong assumptions and also some approximations. Firstly, they consider only the case in which the channel is busy due to a transmission done by an AC in the same station and neglect the presence of other stations in the network. Secondly, they confuse between channel busy probability and collision probability.

3.2.2. Without the Saturation Assumption. In the set of almost all existing analytical models, only few attempts take into consideration the general network conditions case. Engelstad and Osterbo in $[9,21,28,29]$ did a queuing analysis to obtain the queuing and access delays by the use of the z-transform method. The models are based on threedimensional Markov chains in which the first dimension is constant. The proposed model handles virtual collisions but it does not consider the AIFS cooperation with the backoff procedure nor the TXOPLimit. Similarly, Zhang et al. [13] propose a similar three-dimensional Markov chain, where the virtual collision is not handled in the computation of the collision probability. For the first time, Inan et al. [30] introduces the TXOPLimit in the model in which the second dimension in the proposed Markov chain has two significations: it is sometimes equal to the number of packets transmitted during TXOP, and sometimes, it is the backoff counter. TXOP modeling does not need such a complication that renders the whole model ambiguous. Indeed, the complexity of the Markov chain proposed for the model makes it difficult for the authors to trace it completely. Moreover, the model cannot use more than one AC per station because it uses the notion of contention zone-specific collision probabilities. Finally, [31] extends the work of [6] to nonsaturated conditions and it has the same limitations (i.e., it misses the suspension state characteristic, the TXOPLimit differentiation and the used description of AIFS is different from the one described in the standard.

3.3. Summary and Contributions. After this extensive study of the contributions and lacks in the major existing models, we would like to mention that there are other models in the literature that have not been discussed in the above subsections. However, this paper covers the most important ones, as the other models are small derivations of the ones we chose to include in this paper. A summarized comparative study of the main features of the major existing models is illustrated in Table 1. This comparative study can be very helpful to understand how EDCA modeling evolved. Also, it shows the missing features to be completed in order to obtain a more complete analytical model. Such complete model is needed for the design of an accurate admission control algorithm in which it will be the basis. The performed comparison is based on the parameters modeled (CW, AIFS, and TXOPLimit), some baseline features of EDCA (RL: retry limit, B stop: backoff freeze, Int. coll.: internal collision) and performance metrics calculated (Th: throughput, D: access delay).

From this table, we can draw two main conclusions.

(1) Even though almost all EDCA models take into account AIFS and CW differentiation, they unfortunately neglect the use of different TXOPLimit. TXOPLimit differentiation parameter is very important as has been shown in the analysis done in [32]. It was demonstrated that it has noticeable effect on the global performance.

(2) Almost all EDCA models do not consider the modified backoff countdown operation in the 802.11e EDCA accurately. This one should cooperate with the AIFS countdown operation. After each frozen counter in the backoff, the channel must be sensed idle during the whole AIFS period before the backoff countdown procedure starts again. Many of the models previously presented do not reiterate correctly the AIFS countdown before defreezing the backoff counter. Actually, these models which are only two-dimensional Markov chains (one for the backoff stage and one for the backoff counter) could not cover this cooperation process because of this limitation. Therefore, a three-dimensional Markov chain is minimally required.

As a conclusion, all the previous works address only partially the challenges for modeling EDCA. Such partial model may be sufficient if the target is to analyze the effect or the performance characteristics of one parameter taken apart. However, when an accurate analysis of the access delay and achievable throughput is needed or when an analytical model-based admission control algorithm need to be designed, then it is clear that a more complete and accurate model is required. Of course, this one should highlight the exact behavior of the EDCA MAC protocol. Hence, the requirements of such new model are as follows.

(i) The new model should consider the TXOPLimit differentiation parameter accurately. It should highlight the impact of the three differentiation parameters AIFS, CW, and TXOP on the performance of the system.

(ii) The new model should consider the AIFS and backoff cooperation process in EDCA as defined by the standard.

(iii) The new model should capture all QoS specific features for EDCA as described in the standard [1] and model these features correctly. All the aspects of the 
TABLE 1: Comparison of major EDCA models.

\begin{tabular}{|c|c|c|c|c|c|c|c|c|}
\hline & $\mathrm{CW}$ & AIFS & TXOP & $\mathrm{RL}$ & B stop & Int. Coll. & Th & $\mathrm{D}$ \\
\hline [31] & $\checkmark$ & $\sim$ & $\times$ & $\checkmark$ & $\times$ & $\times$ & $\checkmark$ & $\checkmark$ \\
\hline [25] & $\checkmark$ & $\sim$ & $x$ & $\times$ & $\times$ & $x$ & $\checkmark$ & $x$ \\
\hline [21] & $\checkmark$ & $x$ & $\times$ & $\checkmark$ & $\sim$ & $\times$ & $\checkmark$ & $\checkmark$ \\
\hline [6] & $\checkmark$ & $\sim$ & $\times$ & $\times$ & $\times$ & $\times$ & $\checkmark$ & $\checkmark$ \\
\hline [4] & $\checkmark$ & $\sim$ & $\times$ & $\times$ & $\times$ & $\times$ & $\checkmark$ & $\checkmark$ \\
\hline [20] & $\checkmark$ & $\sim$ & $\times$ & $\checkmark$ & $\times$ & $\times$ & $\checkmark$ & $x$ \\
\hline$[30]$ & $\checkmark$ & $\sim$ & $\times$ & $\checkmark$ & $\checkmark$ & $\times$ & $\checkmark$ & $x$ \\
\hline [26] & $\checkmark$ & $\sim$ & $\times$ & $\checkmark$ & $\checkmark$ & $\times$ & $\checkmark$ & $x$ \\
\hline [8] & $\checkmark$ & $\sim$ & $\times$ & $\checkmark$ & $\times$ & $\sim$ & $\checkmark$ & $x$ \\
\hline [22] & $\checkmark$ & $\sim$ & $\times$ & $\checkmark$ & $\checkmark$ & $\sim$ & $\checkmark$ & $\checkmark$ \\
\hline [7] & $\checkmark$ & $\sim$ & $\times$ & $\checkmark$ & $\checkmark$ & $\checkmark$ & $\checkmark$ & $\checkmark$ \\
\hline [27] & $\checkmark$ & $\sim$ & $\times$ & $\checkmark$ & $\checkmark$ & $\checkmark$ & $\checkmark$ & $x$ \\
\hline [24] & $\checkmark$ & $\sim$ & $\times$ & $\checkmark$ & $\checkmark$ & $\checkmark$ & $\checkmark$ & $x$ \\
\hline [23] & $\checkmark$ & $\sim$ & $\times$ & $\checkmark$ & $\checkmark$ & $\checkmark$ & $\checkmark$ & $\checkmark$ \\
\hline [29] & $\checkmark$ & $\sim$ & $\times$ & $\checkmark$ & $\checkmark$ & $\checkmark$ & $\checkmark$ & $\checkmark$ \\
\hline [13] & $\checkmark$ & $\sim$ & $\times$ & $\checkmark$ & $\checkmark$ & $\checkmark$ & $\checkmark$ & $\checkmark$ \\
\hline
\end{tabular}

$\checkmark$ : exists; $\times$ : does not exist; $\sim$ : exists but not accurately.

protocol such as the AIFS countdown procedure, the backoff suspension, the virtual collision between the ACs of the same station, the external collision with other stations, the post-collision, the post-backoff and the retry limit should be present in the model.

(iv) Furthermore, the new model should handle general traffic conditions and should not be limited to the saturation conditions assumption.

\section{Effect of CFB on Transmission Time Modeling}

4.1. Motivations. It was noticeable that the TXOPLimit (i.e., CFB mode) was not modeled in any existing research as the other parameters. Many of the works undertaken to date in the field of 802.11e performance modeling leave this parameter for future works, while others assume that it is automatically present without really considering it. Cited below are some statements that appear in some publications. For example, in [20], authors say: “... TXOP bursting extension is beyond the scope of this paper and is left for future work ...” In [7], it was mentioned: “... In this paper, for simplicity, we only investigate the situation where a station transmits one data frame per TXOP transmission round ..." In [12], the following affirmation is stated: “... Priority based on differentiated Transmission Opportunity (TXOP) limits is not treated explicitly in this paper..." and finally in [33], authors assume that "... One data frame per EDCF-TXOP is transmitted ...”.

This can be considered as a clear gap in these models. Indeed, to be clearer, let us compare the saturated achievable throughput, as it is computed by the model presented in [7], which is a successful one, with the saturated achievable throughput resulting from ns-2 [34] simulations. Obviously, we have the same configuration and parameters in both the model and in the simulations. The unique difference is that the CFB is activated in the simulations, but it is not present in the model, because the model itself did not take it into account.

We clearly see in Figure 1 that there is a big difference between the analytical results and the simulation results when the model does not take into account the TXOPLimit differentiation parameter. For this reason, we are motivated to fill in this gap. Let us show now the effect of this parameter on the performance of 802.11e EDCA.

4.2. Effect of CFB on the Global Performance. To show the effect of the TXOP bursting (i.e., CFB mode) on EDCA performance, a short simulation analysis is conducted. This one is realized using ns-2 [34] enhanced with the TKN's 802.11e implementation [35]. Node topology of the simulation consists of four different wireless QSTAs, contending for channel access, one AP and one wired station. All wireless nodes send their data to the wired node via the AP. Both the AP and these four wireless nodes are situated in the same radio range and distributed as shown in Figure 2. Each QSTA uses all four ACs. Poisson distributed traffic, consisting of 800-bytes packets, was generated at the same rate for each AC. This simulation scenario will be run with different Poisson arrival rates going from $20 \mathrm{~Kb} / \mathrm{s}$ to $1800 \mathrm{~Kb} / \mathrm{s}$ for each $\mathrm{AC}$ at each QSTA, so the total arrival rate in the simulated network varies between $320 \mathrm{~Kb} / \mathrm{s}$ (nonsaturated) and $28800 \mathrm{~Kb} / \mathrm{s}$ (fully saturated). To analyze the effect of the TXOPLimit, we ran the simulation in two different modes. In the first one, CFB was activated, and the TXOPLimit default values are used $(0,0,6.010 \mathrm{~ms}, 3.264 \mathrm{~ms})$, and in the second, it was deactivated and TXOPLimit is set to zero for all ACs.

Figures 3, 4, and 5 show the effect of this differentiation parameter on the throughput and delay performance metrics. It can be clearly seen in Figure 3 that the higher is the TXOPLimit, the higher will be the throughput improvement for the corresponding AC. Here, in this simulation and with 


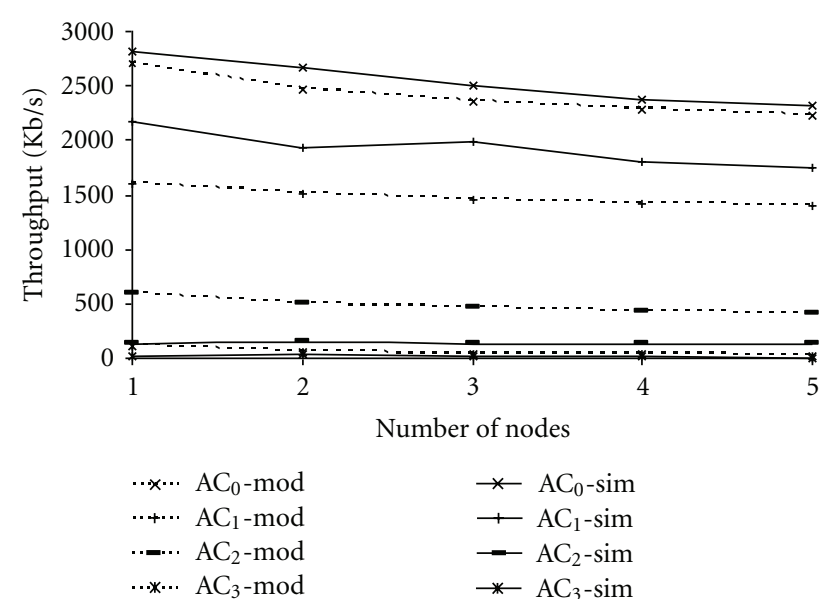

FIGURE 1: Saturated throughput, simulation versus a reference model.

the use of EDCA default parameters, the positive influence of $\mathrm{CFB}$ appears especially on $\mathrm{AC} 1$, which represents the video traffic. It is worth noting that this influence appears not only in the saturation region $(>800 \mathrm{~Kb} / \mathrm{s})$, but also before the saturation (between $200 \mathrm{~Kb} / \mathrm{s}$ and $800 \mathrm{~Kb} / \mathrm{s}$ ).

Furthermore, in CFB, approximately all ACs gain more throughputs as compared to the case in which there is no CFB. The total throughput in the network becomes thus higher, and the medium capacity is well used, this can be clearly seen in Figure 4. This result is explained by the fact that the burst transmission reduces the need for contention and, therefore, the collision probability. This results in an efficient use of the radio channel.

The effect of CFB appears also on the mean access delay of all ACs. Since the examination of the mean access delays for $\mathrm{AC}_{0}$ and $\mathrm{AC}_{1}$ is mostly interesting (the best effort traffic is tolerant to delay), and since these delays are very small compared to those of $\mathrm{AC}_{2}$ and $\mathrm{AC}_{3}$. Figure 5 is drawn with a focus on these delays. The delay of $\mathrm{AC}_{3}$ rapidly reaches high values when approaching the saturation.

In general, while using CFB mode, the mean access delay for all ACs increases this is illustrated in Figure 5. To explain this result, we can say that the burst transmission procedure, forces contending traffics to wait for an additional time, thus increasing the total mean access delay for all ACs.

This analysis shows that the global performance in 802.11 e is clearly different when using TXOP bursting as compared to when not using it. Thus, an accurate modeling for this feature seems important indeed.

4.3. Transmission Time Modeling in CFB Mode. Almost all of the analytical models done for 802.11e EDCA have been constructed in saturation conditions (i.e., they assume that each AC queue has always data to be transmitted). Moreover, in these models, it is assumed that only one data frame is transmitted per transmission round. This assumption taken by all of these models [2-31] makes the models simpler concerning data transmission time. However, such simplification leads to a nonaccurate estimation of the available bandwidth

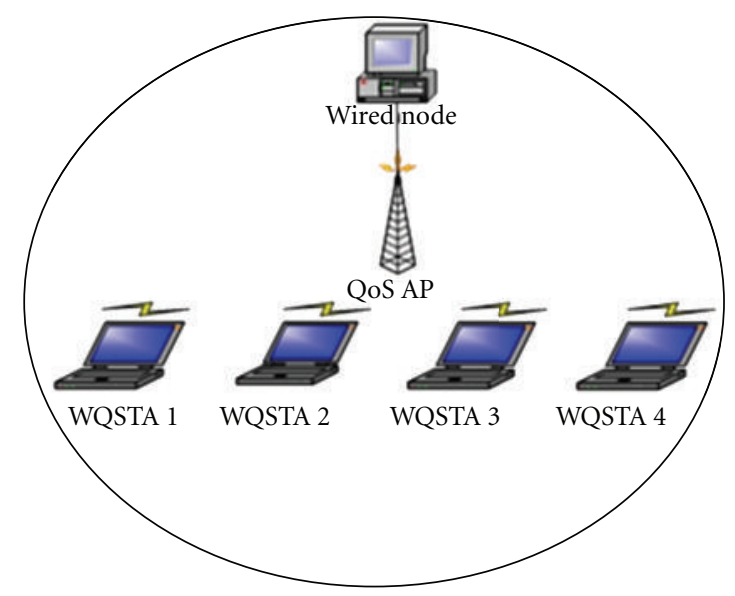

FIgURE 2: Simulation topology.

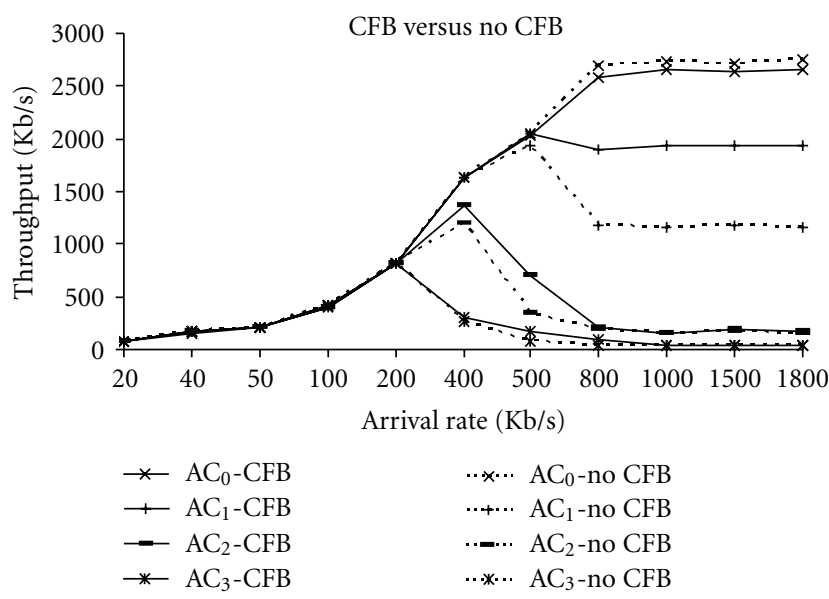

FIgURE 3: Effect of CFB on the achievable throughput per AC.

and access delays while CFB mode is used. Indeed, an $\mathrm{AC}$ may have access to the channel while having only one packet waiting for transmission and it may also access the channel while having a number of packets less or more than the number that can be transmitted in its TXOPLimit. Additionally, in most usual cases, TXOPLimit is not set to zero for all ACs, and it is considered as an important differentiation parameter, giving some ACs more priority to transmit additional data when compared to other ACs. So, the number of frames transmitted may vary according to the queue length for each AC and according to its TXOPLimit. To this variable number of transmitted frames, corresponds a variable transmission time. So, to model the effect of CFB, we need to calculate the correct transmission time.

Here, we aim at calculating the transmission time occupied by a particular $\mathrm{AC}\left(\mathrm{AC}_{i}, i=0 \cdots 3\right)$ when accessing the channel. An $\mathrm{AC}_{i}$ can transmit a set of frames, while the total transmission time does not exceed the TXOPlimit ${ }_{i}$.

Let $P$ be the payload transmission time for a data frame and $H$ the time needed to transmit the header ( $H$ is separated from $P$ in the formulation, because the data rates used for these two quantities are different). According to the access 


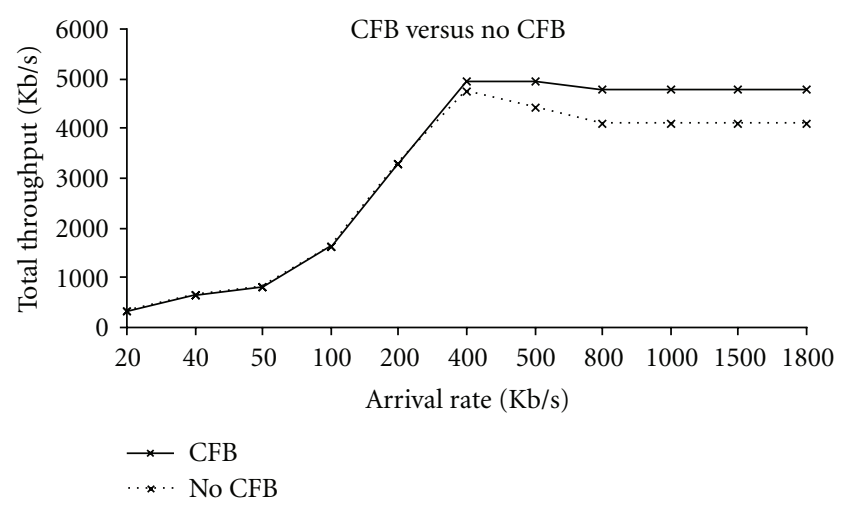

FIgURE 4: Effect of CFB on the total achievable throughput.

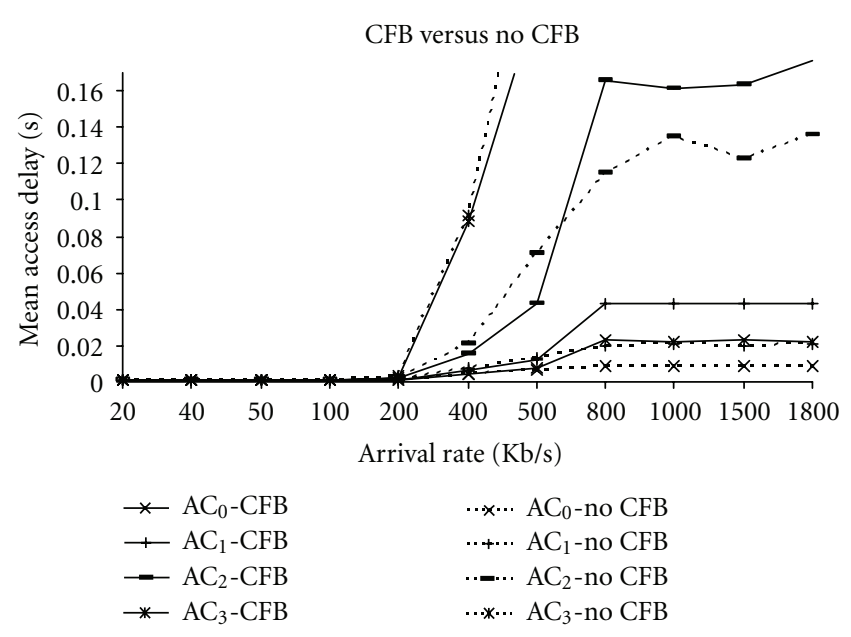

FIgURE 5: Effect of CFB on the access delay per AC.

mode, basic mode or RTS/CTS mode, the transmission time for a single data frame may vary. This time is named $T_{s 1}$. It is given in (1) and (2) and schematized in Figures 6(a) and 6(b). Collision time $T_{c}$ is also illustrated in Figures 6(c) and 6(d) and given in (1) and (2); it will be used later in the analytical model.

In RTS/CTS access mode,

$$
\begin{gathered}
T_{s 1}=\mathrm{RTS}+\mathrm{CTS}+H+P+\mathrm{ACK}+3 \mathrm{SIFS}+4 \delta, \\
T_{c}=\mathrm{RTS}+\mathrm{SIFS}+\delta+\mathrm{CTSTimeout} .
\end{gathered}
$$

In basic access mode,

$$
\begin{gathered}
T_{s 1}=H+P+\mathrm{SIFS}+\mathrm{ACK}+2 \delta, \\
T_{c}=H+p+\mathrm{SIFS}+\delta+\text { ACKTimeout }
\end{gathered}
$$

where $\delta$ is the propagation delay between nodes.

Since frames transmitted during a TXOPLimit ${ }_{i}$ are separated by a SIFS time as illustrated in Figure 7, we may conclude that during this TXOPLimit ${ }_{i}$, an $\mathrm{AC}_{i}$ can transmit a number of frames equal to

$$
N_{\text {TXOP } i}= \begin{cases}{\left[\frac{\text { TXOPLimit }_{i}}{T_{s 1}+\text { SIFS }}\right],} & \text { if } \text { TXOPLimit }_{i} \neq 0, \\ 1, & \text { if } \text { TXOPLimit }_{i}=0 .\end{cases}
$$

$N_{\text {TXOP } i}$ constitutes the maximum number of frames that an $\mathrm{AC}_{i}$ can transmit in its access to the channel. However, the queue of a given $\mathrm{AC}_{i}$ may contain, in some conditions of the arrival rate, a number of frames less than $N_{\mathrm{TXOP} i}$, and in other conditions a greater number. In the first case, it transmits only the existing frames in its queue, while in the second case, it transmits $N_{\text {TXOP } i}$ frames. In the second case, known as the saturation case, the number of frames transmitted can be calculated by (3). But to resolve the problem in the first case (nonsaturation case), we are led to calculate the queue length of the $\mathrm{AC}_{i}$.

In order to calculate the queue length of $\mathrm{AC}_{i}$, we assume an infinite $\mathrm{M} / \mathrm{M} / 1$ queue with a Poisson arrival process of rate $\lambda$ to model the arrival of packets at the MAC buffer. Note that the consideration of this queue model was not done to make assumptions on the service time delay; it is used only to approximate the average number of frames in the queue of each AC. Also, even though the Poisson assumption may not be realistic, it provides insightful results and allows the model to be tractable. According to this assumption, the average number of packets in the system is equal to

$$
N_{\mathrm{Av} i}=\frac{\rho_{i}}{1-\rho_{i}}, \quad \rho_{i}=\lambda_{i} D_{i}, 0 \leq N_{\mathrm{Av} i} \leq \infty .
$$

$D_{i}$ is the mean access delay of $\mathrm{AC}_{i}$ and $\lambda_{i}$ is the mean arrival rate of frames at the $\mathrm{AC}_{i}$ queue.

Now, we can consider that the transmitted frames before the saturation is equal to average number of frames in the queue. So in the general case, the number of frames transmitted in each access to the channel is

$$
N_{\text {Transi }}=\min \left(N_{\mathrm{Av} i}, N_{\mathrm{TXOP} i}\right) .
$$

And the transmission time in a transmission cycle for all ACs can be given by the following equation:

$$
T_{s i}=N_{\text {Transi }}\left(T_{s 1}+\mathrm{SIFS}\right) \quad 0 \leq i \leq 3 .
$$

4.4. Model Validation and Analysis. In order to validate the model proposed for CFB, and to show its usefulness, a set of simulations are performed.

The network topology used to validate the model consists of five different wireless QSTAs, one AP and one wired station. Each QSTA uses all four ACs. Poisson distributed traffic, consisting of 800-bytes packets, was generated at an equal rate for each AC. This simulation scenario will be run with different Poisson arrival rates going from $50 \mathrm{~Kb} / \mathrm{s}$ to $2100 \mathrm{~Kb} / \mathrm{s}$ for each AC at each QSTA. The selected physical protocol for our simulations is $802.11 \mathrm{~b}$. The parameter settings for the $802.11 \mathrm{~b}$ physical layer are depicted in Table 2 . 802.11e parameters, such as AIFSN, CW $\mathrm{CW}_{\min }, \mathrm{CW}_{\max }$, and TXOPLimit, are overridden by the use of 802.11e EDCA default parameters shown in Table 3.

Having a packet size equal to 800 bytes, and using the parameters in Tables 2 and 3, (2) gives $T_{s 1}=1066 \mu \mathrm{s}$. Application of (3) gives the following results: $N_{\mathrm{TXOP} 0}=[3.03]=$ $3, N_{\mathrm{TXOP} 1}=[5.59]=5, N_{\mathrm{TXOP} 2}=N_{\mathrm{TXOP} 3}=1$.

In order to prove the accuracy of the equations proposed, we need to have $D_{i}$. Obtaining $D_{i}$ is not a simple matter. 


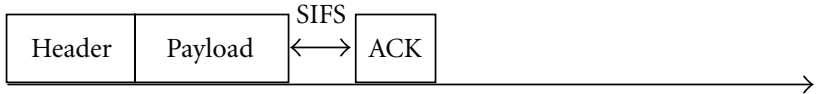

(a) Successful transmission, Basic mode

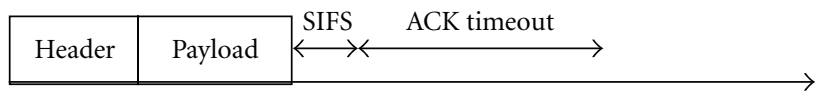

(c) Collision, Basic mode

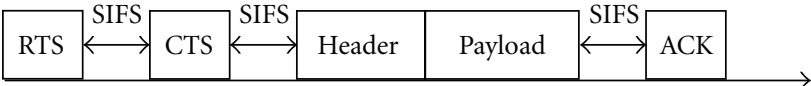

(b) Successful transmission, RTS/CTS mode

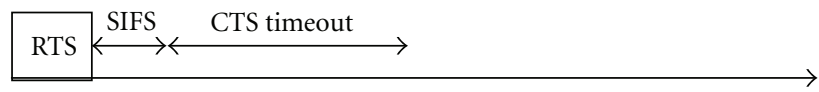

(d) Collision, RTS/CTS mode

Figure 6: Transmission time for one data frame.

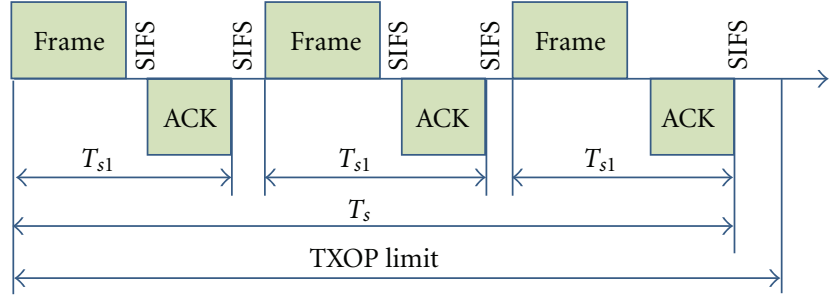

FIgURE 7: Transmission during TXOPLimit.

TABLE 2: 802.11 parameter settings.

\begin{tabular}{lc}
\hline Propagation delay & $64 \mu \mathrm{s}$ \\
Slot time & $20 \mu \mathrm{s}$ \\
SIFS & $10 \mu \mathrm{s}$ \\
Data rate & $11 \mathrm{Mb} / \mathrm{s}$ \\
Basic rate & $2 \mathrm{Mb} / \mathrm{s}$ \\
PLCP data rate & $1 \mathrm{Mb} / \mathrm{s}$ \\
PLCP header length & $48 \mathrm{bits}$ \\
Preamble length & $144 \mathrm{bits}$ \\
Mac header length & $272 \mathrm{bits}$ \\
Ack length & $112 \mathrm{bits}$ \\
\hline
\end{tabular}

TABle 3: Default EDCA parameters.

\begin{tabular}{lcccc}
\hline Priority & 0 & 1 & 2 & 3 \\
\hline AIFSN & 2 & 2 & 3 & 7 \\
CW $_{\min }$ & 7 & 15 & 31 & 31 \\
CW $_{\max }$ & 15 & 31 & 1023 & 1023 \\
TXOPLimit & 3264 & $6016 \mu \mathrm{s}$ & 0 & 0 \\
\hline
\end{tabular}

Indeed, we need to construct a complete analytical model capable of calculating it. Thus, at this stage, and only for validation purposes, before integration to the global model, we will use here delay values obtained from ns-2 simulations. One should note here that once integrated to the global model, this shortcut will obviously not be necessary as $D_{i}$ will also be computed through the model.

Figure 8 proves the accuracy of the proposed approach to calculate the transmission time. In Figure 8, it may be noticed that before the saturation, the average queue length (Q. L.) coincides with the average number of transmissions $(\mathrm{Nb}$. Tr.). This number is less than 1 in average, because most of the time, the queue is empty and there is no frame to be transmitted. While approaching the saturation limit, the queue length starts reaching values greater than $N_{\text {TXOP }}$ for

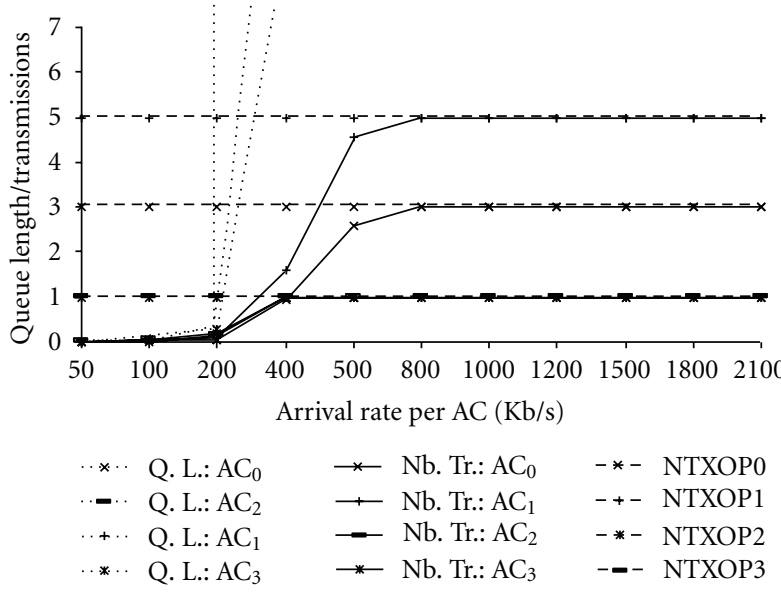

FIGURE 8: Average queue length versus average number of transmissions, for different load rates and for all ACs.

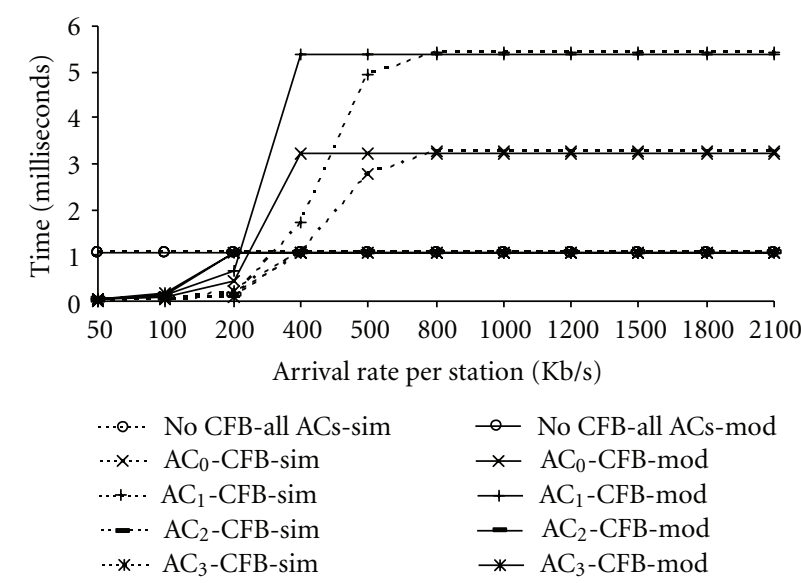

FIGURE 9: Transmission time, previous models, our model, simulation.

each $\mathrm{AC}$ and the average number of transmissions starts to reach the $N_{\text {TXOP }}$. Figure 8 , clearly shows that the $N_{\text {TXOP } i}$ value obtained in the simulation $\left(N_{\mathrm{TXOP} 0}=3, N_{\mathrm{TXOP} 1}=5\right.$ and $\left.N_{\text {TXOP2 }}=N_{\text {TXOP3 }}=1\right)$ is equal to that obtained through our simple model $\left(N_{\mathrm{TXOP} 0}=[3.03]=3, N_{\mathrm{TXOP} 1}=[5.59]=5\right.$ and $\left.N_{\text {TXOP2 }}=N_{\text {TXOP3 }}=1\right)$.

Now, we are looking for validating our model concerning transmission time modeling. This is performed by using our simple model and simulation results.

Figure 9 shows that the average transmission time we got through equations is closely identical to the one we got in the simulation, especially in the saturation conditions, when the 
TXOPLimit limits this transmission time. Details on the simulation and calculation values in nonsaturation conditions and within the saturation limit are discussed and detailed in [32]. Figure 9 also compares between the transmission times while CFB is disabled and while it is not. A large difference between the transmission times in the two modes (with and without CFB) is depicted.

The previous $802.11 \mathrm{e}$ analytical models, which do not take into account TXOPLimit in their modeling, consider a transmission time as being likely equal to $1 \mathrm{~ms}$ whatever is the load rate. With our model, transmission time is not constant all the time. It depends on the load rate. From these results, we can affirm that ignoring $\mathrm{CFB}$ while drawing an analytical model for EDCA is a big limitation for this model, because it cannot predict the correct transmission time as well as the quantity of data transmitted. Therefore, the correct values of throughput and access delay while TXOPLimit differentiation is used.

This transmission time modelling that takes into account the TXOPLimit parameter constitutes a first step towards a complete analytical model for EDCA. In the following sections, we will concentrate on the development of this model.

\section{EDCA Analytical Model in Saturation Conditions}

5.1. Discrete Time Markov Chain Model. In this section, we will present our analytical model developed for saturation conditions. We decided to do that because the saturation conditions constitute the simple way to ignore the queue dynamics and the traffic arrival rate. Also, saturation conditions are used in the most succeeded models in the literature. So, to be able to compare our approach to other ones, we need to work in the same situations. The reader must keep in mind that the model presented here constitutes a particular case of a complete analytical model that will be presented later in this paper and which is mandatory to develop for an efficient admission control algorithm for instance.

It was shown in Section 3. that the introduction of any additional feature of the protocol into the model leads to better accuracy and completeness. So, an in-depth understanding of the protocol behavior is highly recommended to make a complete model. Such a model, incorporating all the features of the protocol is capable of predicting accurately the protocol's behavior for any combination of EDCA parameters.

To be capable of introducing all EDCA features in our model, we will not provide a simple extension of the Bianchi's model as done in other models. This simple extension that considers only the backoff procedure cannot integrate some important features of EDCA that was not present in DCF. So, our approach consists of following the states in which an AC transits during its transmission cycle from the first transmission initiation to the successful transmission or rejection as described in details in Section 2. This approach is the key element that makes our model complete and, therefore, differentiates it from existing models which consider only the backoff states in their Markov chain.
In our model, each state represents an AC in a time slot. At the end of each time slot, an event that triggers a transition to another state occurs. The states that an AC can occupy at a randomly chosen time slot are grouped into six parts (here called periods) representing EDCA operations: AIFS period, backoff period, frozen period, collision period, post collision period, and transmission period.

To represent these periods, we draw a four-dimensional Markov chain for each AC. The first dimension $p(t)$ indicates the period in which the AC is at time $t$. The second dimension $s(t)$ represents the backoff stage and the third one $b(t)$ denotes the value of the backoff counter. Finally, the fourth dimension $r(t)$ indicates the time remaining to leave the current period. The four-dimensional process $(p(t), s(t), b(t)$, and $r(t))$ is supposed to constitute a discrete-time Markov chain under the conditions that the transition probabilities (the collision probability $p_{c}$ and the channel busy probability $p_{b}$ ) are constant.

At each time slot, the state of each AC is determined by $(u, j, k, d): u=A$ stands for AIFS, F for frozen, $B$ for backoff, $C$ for collision, $\mathrm{PC}$ for post-collision and $T$ for transmission. $j=0,1,2 \ldots m ; m$ being the retry limit. $k$ is uniformly chosen from $\left[0, w_{j}\right]$ where $\mathrm{w}_{j}$ depends on the backoff stage and satisfies $w_{j+1}=2 w_{j}+1$ when $w_{j}<w_{\max }\left(w_{0}=w_{\min }\right)$, and $w_{j+1}=w_{j}$ when $w_{j}=w_{\max }$. Finally, $d$ depends on the value of $u$ and will be described in the following.

Herein, we define the specifications of each period apart, the reader is invited to see alternatively, Figures 10 and 11 , to have a graphical illustration of these states and their transitions.

(1) AIFS Period. During this period, the AC must wait for a complete AIFS, while the channel remains idle. For that reason, the AIFS period starts at $(A, j, k,[A]),[A]=$ AIFS[AC]. With probability $1-p_{b}$ (idle channel), the AC transits from $(A, j, k, d)$ to $(A, j, k, d-1)$, while with probability $p_{b}$ (busy channel) it transits from $(A, j, k, d)$ to $(A, j, k,[A])$. So, contrary to other models, if the channel is detected to be busy during the AIFS, the AC rewaits for a complete AIFS before decrementing the backoff which is compliant to the AIFS definition in the standard.

(2) Backoff period. $j=0 \cdots m, k=d=0 \cdots w_{j}$. In any backoff state and during this period, if the channel is busy $\left(p_{b}\right)$, the AC transits to the frozen period conserving the same $j$ and $k$ values. When $k$ (backoff counter) reaches 0 , there is automatically a transmission attempt. With $p_{c}$ probability, the AC transits to a collision period incrementing $j$ by one otherwise, it transits to the transmission period. After the retry limit in state $(B, m, 0$, and 0$)$ and in case of collision, we have a rejection and a transition to the first AIFS period takes place in order to make a transmission of the next frame.

(3) Frozen Period. $j$ and $k$ remains constant during frozen states while $d$ decrements by one at each time slot. Here, $d$ is the time remaining before the backoff counter is reactivated, its initial value is equal to $[N]$. $[N]$ is the average frozen time, which is equal to the average transmission time of all ACs.

(4) Collision Period. $k=0, d$ starts at $\left[T_{c}\right]$. This is the average time needed to detect a collision. After the collision, 


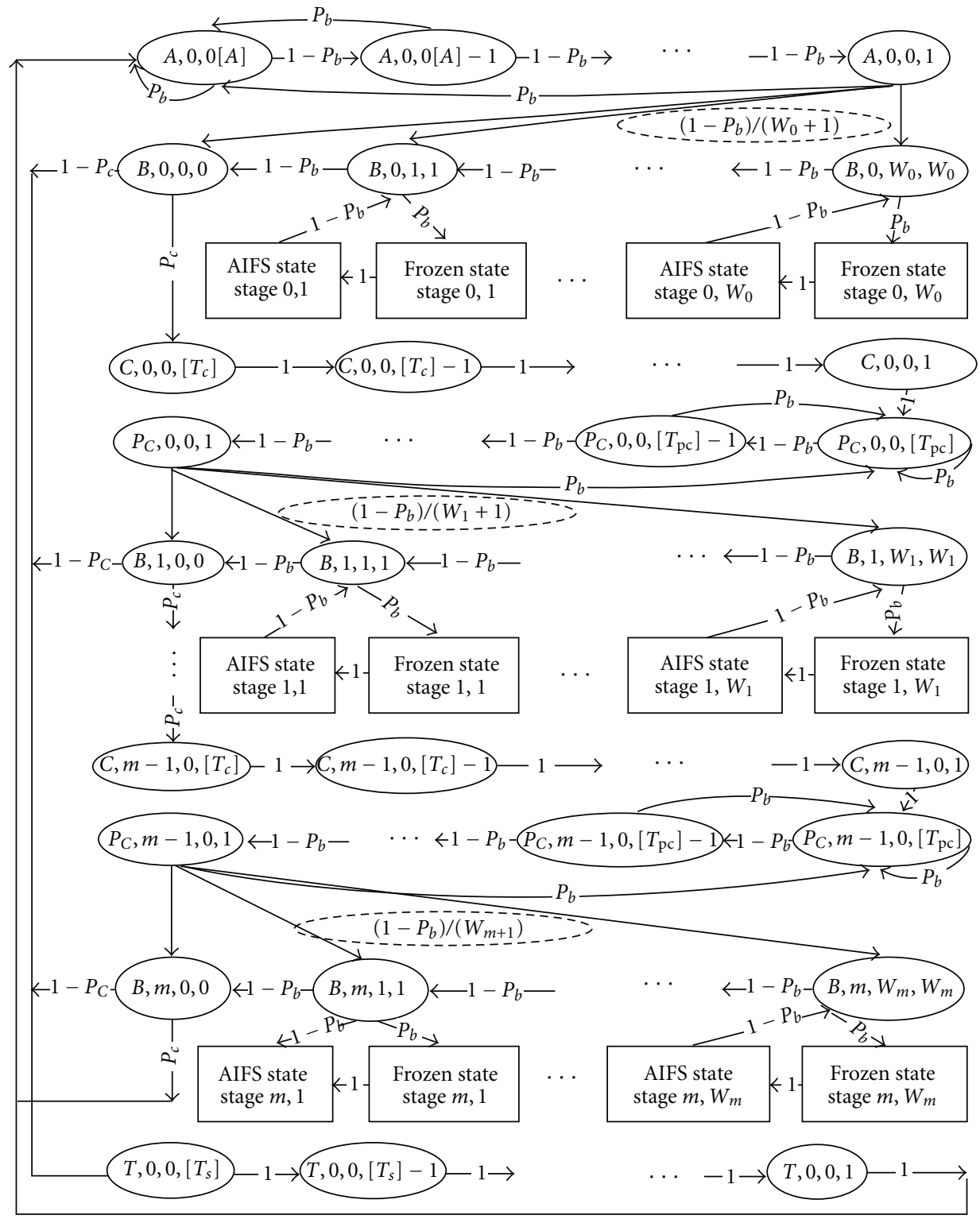

FIGURE 10: Markov chain of the model.

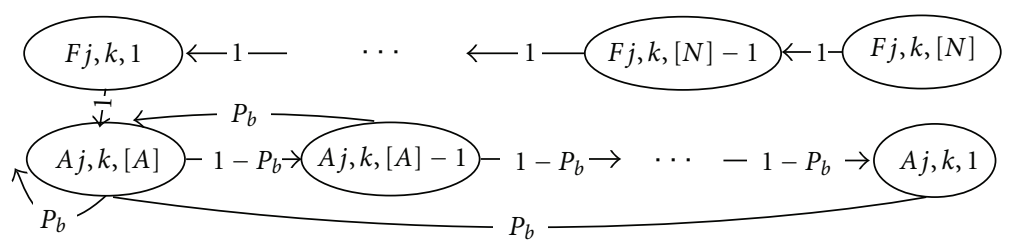

Figure 11: Frozen and AIFS blocs $(j, k) ; 0 \leq j \leq m, 1 \leq k \leq w_{j}$.

the AC transits to a post collision state and reenters again the backoff at stage $j+1$.

(5) Post Collision Period. $k=0, d$ starts at $\left[T_{\mathrm{pc}}\right]$. This is the post collision waiting time. It is equal here to AIFS [AC] because the $\mathrm{AC}$ is involved in the collision.

(6) Transmission Period. $j=k=0, d=\left[T_{s}\right] \cdots 1$. Here, the AC begins its transmission and continues until its queue becomes empty or until the expiry of its TXOPLimit [AC]. $\left[T_{s}\right]$ is the average transmission time, it is calculated in Section 4.

5.2. System Equations. To resolve this Markov chain system, we need to calculate the probability of each state by applying the global balance equations. For that aim, we consider $P_{i, j, k, d}$ the steady state probability of a given state $(i, j, k$, and $d)$. 
The probability of this state is equal to the sum of all transition probabilities that lead to it. So, let us first assume that $P_{B, 0,0,0}$ is known and let us calculate all the other state probabilities with respect to it. Although our model is a four-dimensional one, the development of the global balance equations produces the following set of simple equations:

$$
\begin{gathered}
P_{B, j, 0,0}=p_{c}^{j} P_{B, 0,0,0}, \quad 0 \leq j \leq m, \\
P_{B, j, k, k}=\frac{w_{j}-k+1}{w_{j}+1} \frac{P_{B, j, 0,0}}{1-p_{b}}, \quad 0 \leq j \leq m, 1 \leq k \leq w_{j}, \\
P_{C, j, 0, d}=p_{c} P_{B, j, 0,0}, \quad 0 \leq j \leq m-1,1 \leq d \leq\left[T_{c}\right], \\
P_{P C, j, 0, d}=\frac{p_{c}}{\left(1-p_{b}\right)^{d}} P_{B, j, 0,0}, \\
0 \leq j \leq m-1,1 \leq d \leq\left[T_{\mathrm{pc}}\right] \\
P_{F, j, k, d}=\frac{w_{j}-k+1}{w_{j}+1} \frac{p_{b}}{1-p_{b}} P_{B, j, 0,0}, \\
0 \leq j \leq m, 1 \leq k \leq w_{j}, 1 \leq d \leq[N], \\
P_{A, j, k, d}=\frac{w_{j}-k+1}{w_{j}+1} \frac{p_{b}}{\left(1-p_{b}\right)^{d+1}} P_{B, j, 0,0}, \\
0 \leq j \leq m, 1 \leq k \leq w_{j}, \quad 1 \leq d \leq[A], \\
P_{A, 0,0,0, d}=\frac{\left(1-p_{c}^{m+1}\right) P_{B, 0,0,0}, \quad 1 \leq d \leq\left[T_{s}\right] .}{\left(1-p_{b}\right)^{d}} P_{B, 0,0,0}, \quad 1 \leq d \leq[A],
\end{gathered}
$$

Now, to get $P_{B, 0,0,0}$, we have to resolve the normalization condition: $\sum_{i, j, k, l} P_{i, j, k, l}=1$ which gives (15)

$P_{B, 0,0,0}$

$$
=\left[\begin{array}{c}
\frac{1}{p_{b}}\left(\frac{1}{\left(1-p_{b}\right)^{[A]}}-1\right)+\left(1-p_{c}^{m+1}\right)\left(\left[T_{s}\right]+\frac{1}{1-p_{c}}\right) \\
+p_{c} \frac{\left(1-p_{c}^{m}\right)}{\left(1-p_{c}\right)}\left(\left[T_{c}\right]+\frac{1}{p_{b}}\left(\frac{1}{\left(1-p_{b}\right)^{\left[T_{\mathrm{pc}}\right]}}-1\right)\right) \\
+\frac{1}{2\left(1-p_{b}\right)}\left([N] p_{b}+\frac{1}{\left(1-p_{b}\right)^{[A]}}\right) \sum_{j=0}^{m} p_{c}^{j} w_{j}
\end{array}\right]^{-1} .
$$

Once $\left[T_{s}\right],\left[T_{c}\right],\left[T_{\mathrm{pc}}\right],[A],[N], m, w_{j}, p_{c}$, and $p_{b}$ are known, all state probabilities can be obtained using (7)-(15). $\left[T_{\mathrm{pc}}\right],[A],[N], m$, and $w_{j}$ are defined in Section 5.1, $\left[T_{s}\right]$ and $\left[T_{c}\right]$ are defined in Section 6.2, the key issue is then to calculate $p_{c}$ and $p_{b}$.

To calculate $p_{c}$ and $p_{b}$, let us see how a collision may occur, and when the channel is considered as busy. Let $\tau_{i}$ be the probability for an $\mathrm{AC}_{i}$ attempt to access the channel in a random time slot. According to our model, $\tau_{i}$ is the sum of all probabilities of backoff states with a counter equal to zero.

$$
\tau_{i}=\sum_{j=0}^{m} P_{B, j, 0,0}=\frac{1-p_{c i}^{m+1}}{1-p_{c i}} P_{B, 0,0,0}, \quad 0 \leq i \leq 3 .
$$

To match the exact behavior of EDCA, we firstly get the value from the viewpoint of an $\mathrm{AC}$ then from the viewpoint of a station while following the correct behavior of the protocol. Moreover, we differentiate between virtual and external collisions in order to obtain more accurate values. Without loss of generality, we assume a fixed number of active stations $M$ in the same radio range; each station has its four ACs active.

From the viewpoint of a station, the probability $\tau$ that it accesses the channel is the probability that at least one of its ACs tries to access the channel:

$$
\tau=1-\prod_{i=0}^{3}\left(1-\tau_{i}\right)
$$

The Internal Collision Probability. For $\mathrm{AC}_{i}$, the probability is that at least one of the higher priority ACs tries to access the channel simultaneously with it, so

$$
P_{\text {cint }_{i}}=1-\prod_{j>i}\left(1-\tau_{j}\right) .
$$

The External Collision Probability. The probability is that at least one of the other $(M-1)$ stations tries to access the channel at the same time slot

$$
P_{c \text { ext }_{i}}=1-(1-\tau)^{M-1} .
$$

Thus, an $\mathrm{AC}_{i}$ is free from collision only when neither other higher priority ACs, nor another station is trying to access the channel. So, the total collision probability is given by

$$
P_{c i}=1-(1-\tau)^{M-1} \prod_{j>i}\left(1-\tau_{j}\right) .
$$

For the busy channel probability, let $v_{i}$ be the probability for the channel to be occupied by an $\mathrm{AC}_{i}$, this is the probability for it to be in transmission or in an external collision state

$$
v_{i}=\left(\left[T_{s i}\right]\left(1-p_{c i}^{m+1}\right)+\left[T_{c i}\right] \frac{p_{c i}-p_{c i}^{m+1}}{1-p_{c i}} p_{c e x t_{i}}\right) P_{B, 0,0,0} .
$$

The probability for the channel to be occupied by a station is the probability for it to be occupied by one and only one $\mathrm{AC}$ from this station, so

$$
v=\sum_{i=0}^{3} v_{i} \prod_{j \neq i}\left(1-v_{j}\right) .
$$


Finally, the channel is considered as idle if none of the stations is using it. Thus,

$$
P_{b}=1-(1-v)^{M} .
$$

To summarize, the overall system form a nonlinear equations system with three equations ((15), (20), and (23)) with only three unknown variables; $p_{b}, p_{c}$, and $P_{B, 0,0,0}$. It can be easily solved by means of numerical methods. Once resolved, the channel busy probability $p_{b}$, the collision probability for each $\mathrm{AC} p_{c i}$ and the probability of being in the $(B, 0,0,0)$ state $P_{B, 0,0,0}$ become known. They constitute the key variables in the throughput and access delay calculation as we will see in the following subsection.

5.3. Throughput Analysis. Let $S_{i}$ be the normalized system throughput of $\mathrm{AC}_{i}$.

$$
S_{i}=\frac{E\left[\mathrm{AC}_{i} \text { payload data successfully transmitted }\right]}{E[\text { Interval between } 2 \text { successive transmissions }]} .
$$

The probability $p_{s i}$ for a transmission of $\mathrm{AC}_{i}$ to be successful is equal to the probability for exactly one $\mathrm{AC}_{i}$ in only one station to be transmitted, so

$$
P_{s i}=M p_{t i}(1-v)^{M-1} \prod_{j>i}\left(1-v_{j}\right),
$$

where $p_{t i}$ is

$$
P_{t i}=\sum_{d=1}^{\left[T_{s}\right]} p_{T, 0,0, d}
$$

In our model, contrary to all other models, the TXOPLimit parameter is taken into account. As we assume here saturation conditions, we suppose that the AC's queue contains sufficient data to be transmitted during its TXOPLimit. So, as explained in Section 4, the number of frames that can be transmitted by $\mathrm{AC}_{i}$ during its access to the channel is equal to $N_{\mathrm{TXOP} i}$ defined by (3) and the time needed to transmit these $N_{\mathrm{TXOP} i}$ frames is then obtained using (5) and (6)

$$
T_{s i}=N_{\mathrm{TXOP} i}\left(T_{s 1}+\mathrm{SIFS}\right) .
$$

$E[P]$ being the average packet payload size, the average amount of payload information successfully transmitted is $P_{s i} E[P] N_{\mathrm{TXOP} i}$. The average time interval in time slots between two consecutive transmissions is obtained considering that with probability $\left(1-p_{b}\right)$, the channel is idle with probability $p_{b} p_{s i}$, it contains a successful transmission, and with probability $p_{b}\left(1-p_{s i}\right)$, it contains a collision. Therefore, (24) becomes

$$
S_{i}=\frac{p_{s i} E[P] N_{\mathrm{TXOP} i}}{\left(1-p_{b}\right)+p_{b} \sum_{j=0}^{3} p_{s j} T_{s i}+p_{b}\left(1-\sum_{j=0}^{3} p_{s j}\right) T_{c i}} .
$$

So, (28) gives the saturated throughput of $\mathrm{AC}_{i}$. One should note here that a single equation is needed to calculate the throughput per AC. So, once the nonlinear system is resolved as explained earlier, the throughput can be calculated without any computational complexity.

5.4. Access Delay Analysis. We introduced the fourth dimension in our Markov chain to calculate the access delay per $\mathrm{AC}$, This dimension, containing the time necessary to leave a given period, a simple recursive method permits us to calculate the average time between the starting point in a transmission attempt (first AIFS state) and the successful transmission (transmission state). This time constitutes the mean access delay in EDCA.

Let $D_{i, j, k, d}$ be the time delay from the state $(i, j, k$, and $d)$ to the successful transmission. The resolution of our system gives a set of equations in chain. So, we can use $D_{B, m, 0,0}$ to calculate the delay of all states in the following order.

$D_{B, j, 0,0}$ given, (29) gives the backoff states delays of the stage $j$. At the same time, we can calculate the AIFS and frozen states delays of the same stage $j$ by using (30) and (31)

$$
\begin{aligned}
D_{B, j, k, k}= & D_{B, j, k-1, k-1}+\frac{1+[N] p_{b}\left(1-p_{b}\right)^{A}}{\left(1-p_{b}\right)^{A+1}}, \\
& 0 \leq j \leq m, 1 \leq k \leq w_{j}, \\
D_{A, j, k, 1}= & D_{B, j, k, k}+\frac{1}{\left(1-p_{b}\right)^{A}}, \\
& 2 \leq d \leq[A], 0 \leq j \leq m, 1 \leq k \leq w_{j}, \\
D_{A, j, k, d}= & D_{A, j, k, d-1}+\frac{1}{\left(1-p_{b}\right)^{A-(d-1)}}, \\
& 0 \leq j \leq m, 1 \leq k \leq w_{j}, 2 \leq d \leq A, \\
D_{F, j, k, d}= & D_{A, j, k, A}+d, \\
& 0 \leq j \leq m, 1 \leq k \leq w_{j}, 1 \leq d \leq[N] .
\end{aligned}
$$

To pass from one stage to another one, (32), (33), and (34) must be used. Equation (34) allows us to finally obtain $D_{B, j, 0,0}$ as needed in the equations above

$$
\begin{aligned}
& D_{P C, j, 0,1}= \frac{\sum_{k=0}^{w_{j+1}} D_{B, j+1, k, k}}{w_{j+1}+1}+\frac{1}{\left(1-p_{b}\right)^{\left[T_{\mathrm{pc}]}\right.},} \\
& 0 \leq j \leq m-1, \\
& D_{P C, j, 0, d}= D_{\mathrm{pc}, j, 0, d-1}+\frac{1}{\left(1-p_{b}\right)^{\left[T_{\mathrm{pc}}\right]-(d-1)},} \\
& 0 \leq j \leq m-1,2 \leq d \leq\left[T_{\mathrm{pc}}\right], \\
& D_{C, j, 0, d}= D_{P C, j, 0,\left[T_{\mathrm{pc}}\right]}+d, \\
& 0 \leq j \leq m-1,1 \leq d \leq\left[T_{c}\right], \\
& D_{B, j, 0,0}= p_{c} D_{C, j, j,\left[T_{c}\right]}+\left(1-p_{c}\right), \quad 0 \leq j \leq m-1 .
\end{aligned}
$$


Finally, to calculate the delays of the first AIFS period, (35) is to be used

$$
\begin{gathered}
D_{A, 0,0,1}=\frac{\sum_{k=0}^{w_{0}} D_{B, 0, k, k}}{w_{0}+1}+\frac{1}{\left(1-p_{b}\right)^{[A]}}, \\
D_{A, 0,0, d}=D_{A, 0,0, d-1}+\frac{1}{\left(1-p_{b}\right)^{[A]-(d-1)}}, \quad 2 \leq d \leq[A] .
\end{gathered}
$$

For the whole Markov chain, once $D_{B, m, 0,0}$ is known, the mean access delay of the system which is by definition equal to $D=D_{A, 0,0,[A]}$ can be calculated using the set of (27)-(35). The mathematical development and calculation of these equations permits us to obtain finally the following unique equation for the computation of the mean access delay per AC

$$
\begin{aligned}
D_{i}= & 1+\frac{1+[N] p_{b}\left(1-p_{b}\right)^{A}}{\left(1-p_{b}\right)^{A+1}} \sum_{i=0}^{m} p_{c i}^{i} w_{i}+\frac{1-\left(1-p_{b}\right)^{A}}{p_{b}\left(1-p_{b}\right)^{A}} \\
& +p_{c i} \frac{1-p_{c i}^{m}}{1-p_{c i}}\left(\frac{1-\left(1-p_{b}\right)^{\left[T_{\mathrm{pc}}\right]}}{p_{b}\left(1-p_{b}\right)^{\left[T_{\mathrm{pc}}\right]}}+\left[T_{c}\right]\right) .
\end{aligned}
$$

So, (36) gives the saturated access delay of $\mathrm{AC}_{i}$. We note here, as in the case of throughput calculation, that this unique equation can be applied to calculate the mean access delay per AC. So, the delay calculation involves no computational overhead also. The complexity of our model resides only in the nonlinear system resolution.

Being able to calculate the throughput and the delay for the four ACs by the use of only two mathematical equations is a very interesting result of our model.

\section{Saturation Model Validation}

To validate our model in saturation conditions, we have implemented it in Matlab. Also, and in order to situate our model with other ones, we have chosen a successful one from the literature [7] and implemented it in Matlab also. This model [7] is selected in our comparison, because it is one of the most complete models according to our previous comparison in Table 1. This is the model that incorporates the most important number of features compared to other models (different AIFS, different CW, and external and virtual collisions). This model is noted as the "reference model" in this section. The selected physical protocol for validations is $802.11 \mathrm{~b}$. 802.11e EDCA default parameters are used, and these physical and MAC parameters are already defined in Tables 2 and 3 . To decide about the validity of these two models, we compared them to simulation results.

The simulation topology consists of a variable number of wireless QSTAs, going from 2 to 10, all nodes are situated in the same radio range. Each QSTA uses all four ACs. Poisson distributed traffic, consisting of 800-bytes packets, was generated at equal amounts in each AC with an arrival rate equal to $2 \mathrm{Mb} / \mathrm{s}$. These values are chosen in order to

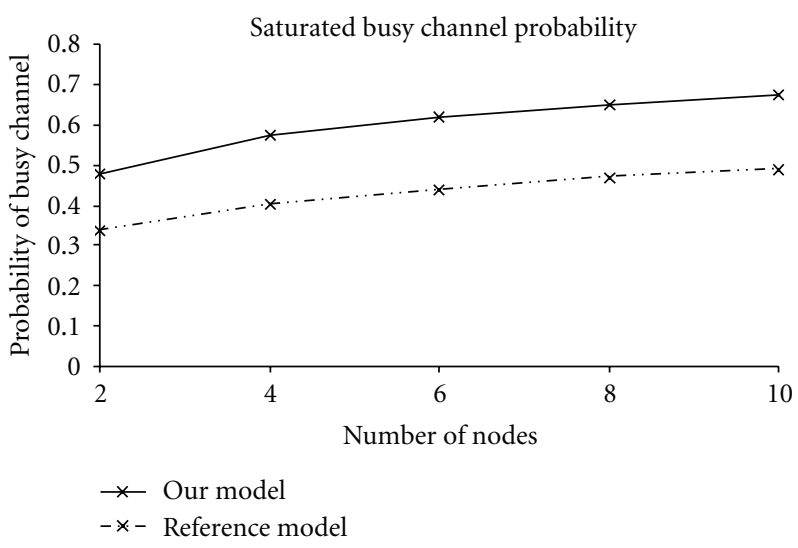

FIGURE 12: Probability of busy channel, basic mode.

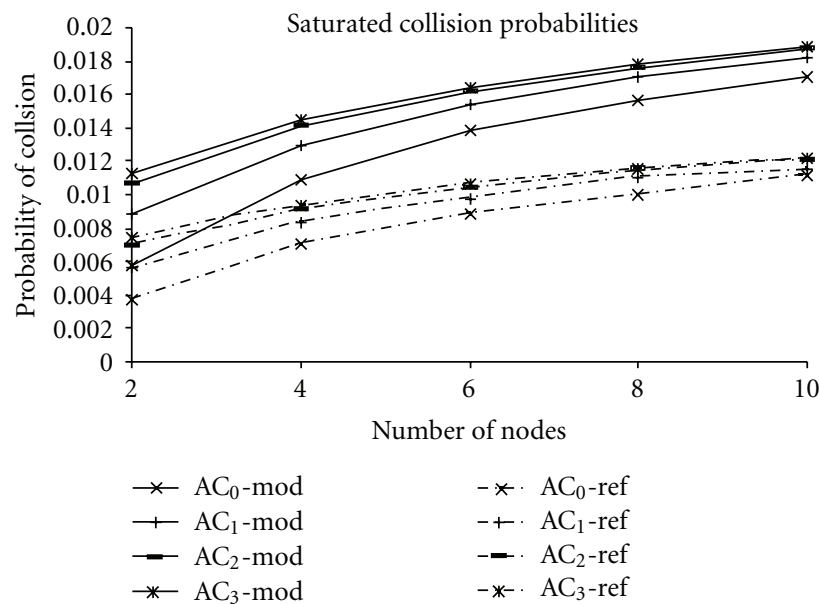

FIgURE 13: Probability of collision, basic mode.

ensure that we are working under saturation conditions in the simulation scenario (in case of two stations only, we have a total offered load equal to $16 \mathrm{Mb} / \mathrm{s}$ which is larger than the $802.11 \mathrm{~b} 11 \mathrm{Mb} / \mathrm{s}$ ). In our model, as well as in the simulation, we run the program with and without TXOPLimit differentiation to show the impact of the use of this parameter on the performance and to assess the validity of our proposed model regarding the CFB modeling. One should also note that the reference model does not allow us to test CFB. This feature is not modeled in it at all.

6.1. Transition Probabilities. First of all, let us see the channel busy probability and the collision probability as obtained in the modeling analysis without TXOP. It can be seen in Figures 12 and 13 that these two probabilities increase when the number of active stations increases. Differentiation between ACs is very clear in Figure 13; the AC of higher priority has the lower collision probability.

What is important to note here is that the busy channel probability in the reference model is always between 0.3 and 0.5 . This one is less than that of our model which is between 0.5 and 0.7 . Logically, this probability must have higher values when the system works under saturation conditions, 


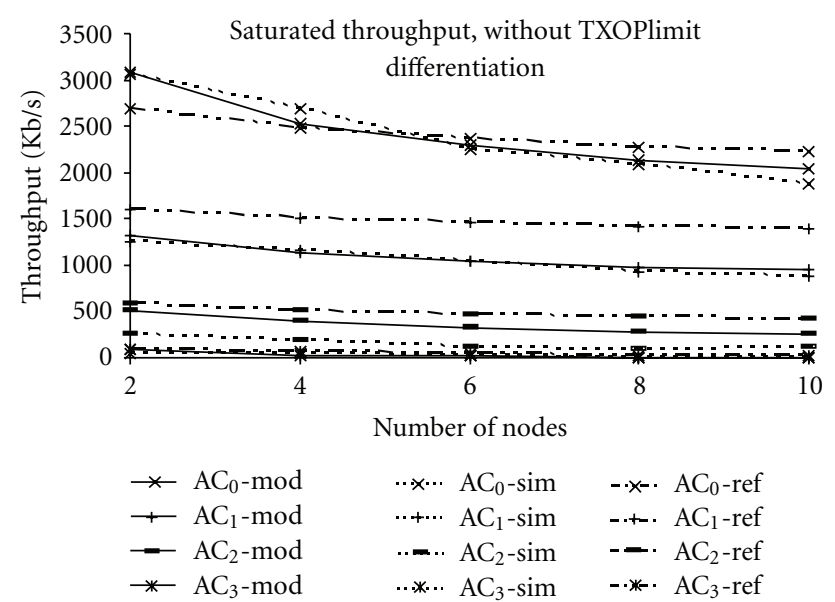

Figure 14: Throughput per AC, without TXOP.

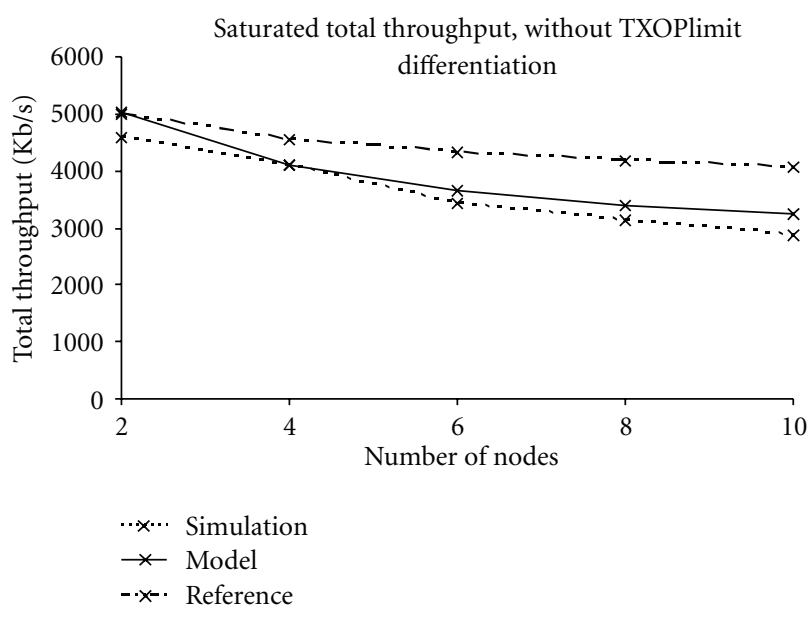

FIgURE 15: Total throughput, without TXOP.

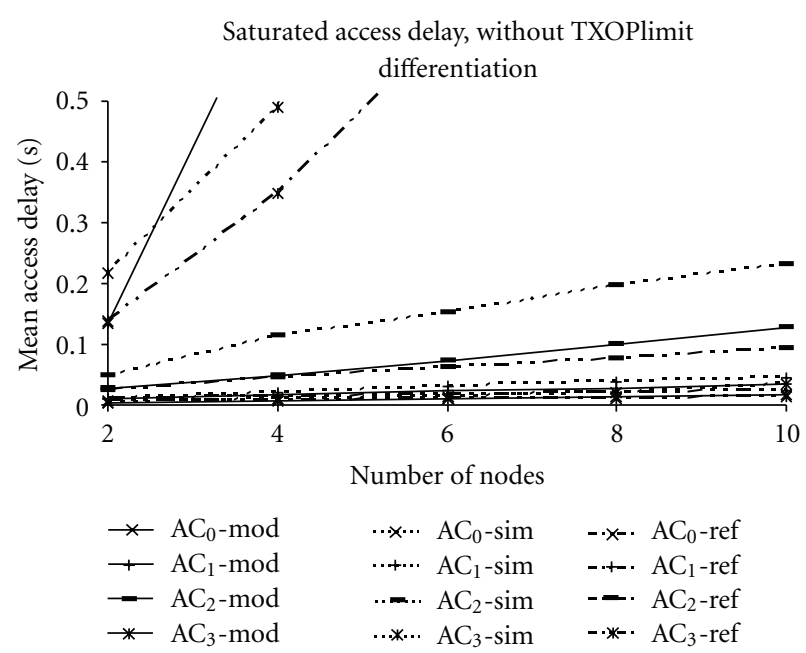

FIGURE 16: Mean access delay per AC, without TXOP. as the channel must be busy almost all the time. The results obtained in the reference model do not seem logical when looking at the collision probability: low collision probability values must lead to high channel busy probability values. The probability values obtained using our model, are more realistic.

6.2. Performance Metrics without TXOPLimit. Now, to assess the accuracy of our model, let us compare the performance metrics obtained by our model as compared to the reference model and to simulation results.

Figure 14 through Figure 16 depict these results for the basic mode, while TXOP is not used in the two models, so this comparison concerns other aspects of the protocol modeling. The results show that the performance of our model is closer to the simulation than those of the reference model. This is verified for all ACs. This proves that our approach in the development of our model gives more accurate results than others. These results can be justified by the underlying reasoning, analysis, calculation, and norm appliance. This is not the case neither for the reference model nor for the other models presented in the literature.

Figure 14 through Figure 16 show that even under saturated network conditions, the value of the throughput and delay does not remain constant when changing the number of stations. When the number of stations increases, the throughput of each AC decreases the total achievable throughput in the network decreases, and the access delay increases. Adding more active stations to the same radio range leads to more and more contention and a higher collision probability, and this leads to a network capacity loss.

6.3. Performance Metrics with TXOPLimit. Now, let us see the validity of our model in modeling the TXOPLimit. In Figures 17, 18, and 19, the achievable throughput of each $\mathrm{AC}$, the total achievable throughput and the access delay are respectively drawn in case where CFB is activated. These figures clearly show that the analytical results match exactly the simulation results.

If we compare Figures 14, 15, and 16, to Figures 17, 18, and 19, we can depict that the results we get in Section 4 are verified again here, by our analytical model: TXOPLimit differentiation parameter privileges the $\mathrm{AC}_{1}$ over low priority ACs, the total achievable throughput is greater than that obtained where $\mathrm{CFB}$ is deactivated, and the mean access delay increases with the activation of CFB. So, the importance of including this parameter in the protocol behavior, which is demonstrated in Section 4, is validated here analytically. Our model is the first one to include the modeling of this feature accurately. Figures 17, 18, and 19 show that our model does it and that our analytical calculation gives very satisfactory results. This is one of our major contributions.

\section{Model Extension to General Conditions}

7.1. Markov Chain Extension. As we said before, the saturation condition constitutes only a particular case of a complete analytical model. This complete analytical model is necessary 


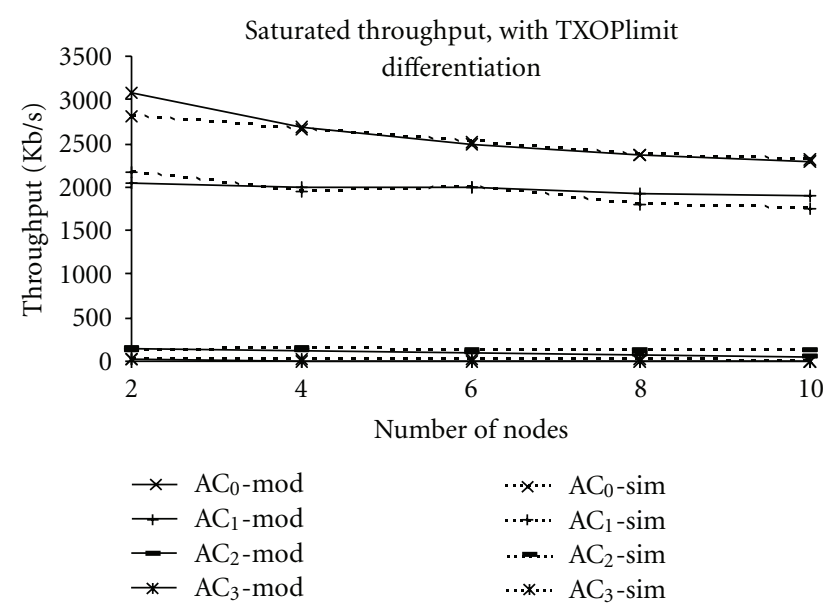

FIgURE 17: Throughput per AC, with TXOP.

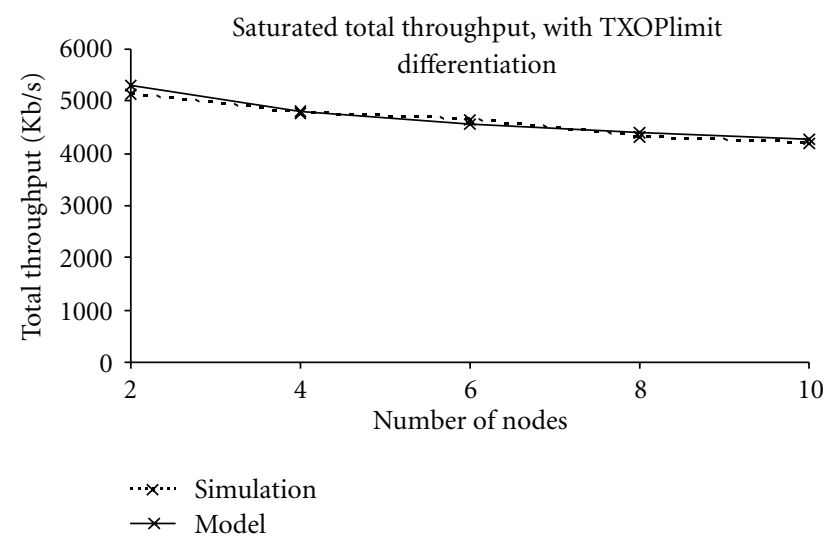

FIgURe 18: Total throughput, with TXOP.

for an efficient admission control. Because our main objective is to obtain an accurate analytical model for the use in an admission control algorithm (which in turn aims to avoid the network to reach a high saturation state), we will extend the proposed model in Section 5 to general conditions. To extend this model to the case of general traffic conditions, going from extremely nonsaturated $\mathrm{AC}$ queues to extremely saturated $\mathrm{AC}$ queues, it is obviously necessary to extend the Markov chain. Our approach while doing these extensions consists of following the states an AC can occupy after the completion of the last transmission while its queue is empty.

In fact, as mentioned in the standard [1], after each successful transmission, the transmitting AC initiates another random backoff, even if there is no other pending frames to be delivered. This is often referred to as post-backoff, as this is done after, not before, a transmission.

So, in our complete Markov chain, after each transmission, there is always a transition from state $(T, 0,0$, and 1$)$ to state $(A, 0,0$ and $[A])$ to accomplish the post-backoff procedure. This is a normal first stage backoff, where the AC waits for an AIFS time and chooses a random backoff to decrement the backoff counter until it reaches the state $(B, 0,0$ and 0$)$. We do not care what may happen during this

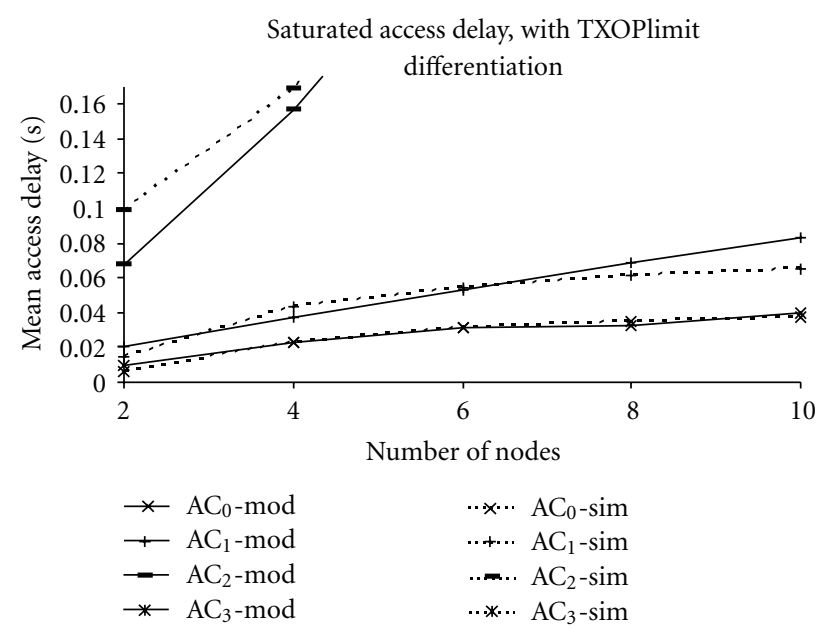

FIgUre 19: Mean access delay per AC, with TXOP.

post backoff procedure at the level of the AC queue, because this procedure will be accomplished totally whatever is the queue state. However, it is very important to see what may happen once the $\mathrm{AC}$ reaches the state $(B, 0,0$ and 0$)$. Here, the AC does not try to access the channel immediately as in the saturation conditions. The transition from this state depends on the queue state. If there is data to be transmitted in the queue at this moment, it attempts directly to access the channel. If the queue is empty, the AC transits to an idle state in which it waits for the arrival of new packets. So, our Markov chain is extended by the addition of new idle states $(I, 0,0$, and $d), 1 \leq d \leq[W]$, where $[W]$ is the average waiting time with an empty queue.

Let $p_{e}$ be the probability of having an empty queue after the completion of the last transmission and the post backoff. The probability of transition from state $(B, 0,0$, and 0$)$ to $(I, 0,0$, and 0$)$ is then equal to $p_{e}$, the probability of transition from state $(B, 0,0$, and 0$)$ to $\left(T, 0,0\right.$, and $\left.\left[T_{s}\right]\right)$ is $\left(1-p_{e}\right)^{*}\left(1-p_{c}\right)$ and finally the probability of transition from $(B, 0,0$, and 0$)$ to $(C, 0,0$, and $[T C])$ is equal to is $\left(1-p_{e}\right)^{*} p_{c}$.

After the expiry of the average waiting time, the queue is not considered empty and there is a transmission attempt. With probability $\left(1-p_{c}\right)$ the transmission succeeds and with probability $p_{c}$ there is a collision. In this latest case another first stage backoff is to be done and the process continues normally. The new states introduced and the corresponding transition probabilities are shown in Figure 20.

7.2. System Equations. The resolution of the new Markov chain according to the global balance equations gives the following new state probabilities. The other state probabilities $((8)-(13))$, given in Section 5 remain unchanged

$$
\begin{gathered}
P_{B, j, 0,0}=\left(1-p_{e}\right) p_{c}^{j} P_{B, 0,0,0} \quad 1 \leq j \leq m, \\
P_{T, 0,0, d}=\left(1-p_{c}^{m+1}\left(1-p_{e}\right)-p_{c} p_{e}\right) P_{B, 0,0,0} \quad 1 \leq d \leq\left[T_{s}\right],
\end{gathered}
$$

$$
P_{I, 0,0, d}=p_{e} P_{B, 0,0,0} \quad 1 \leq d \leq[W],
$$




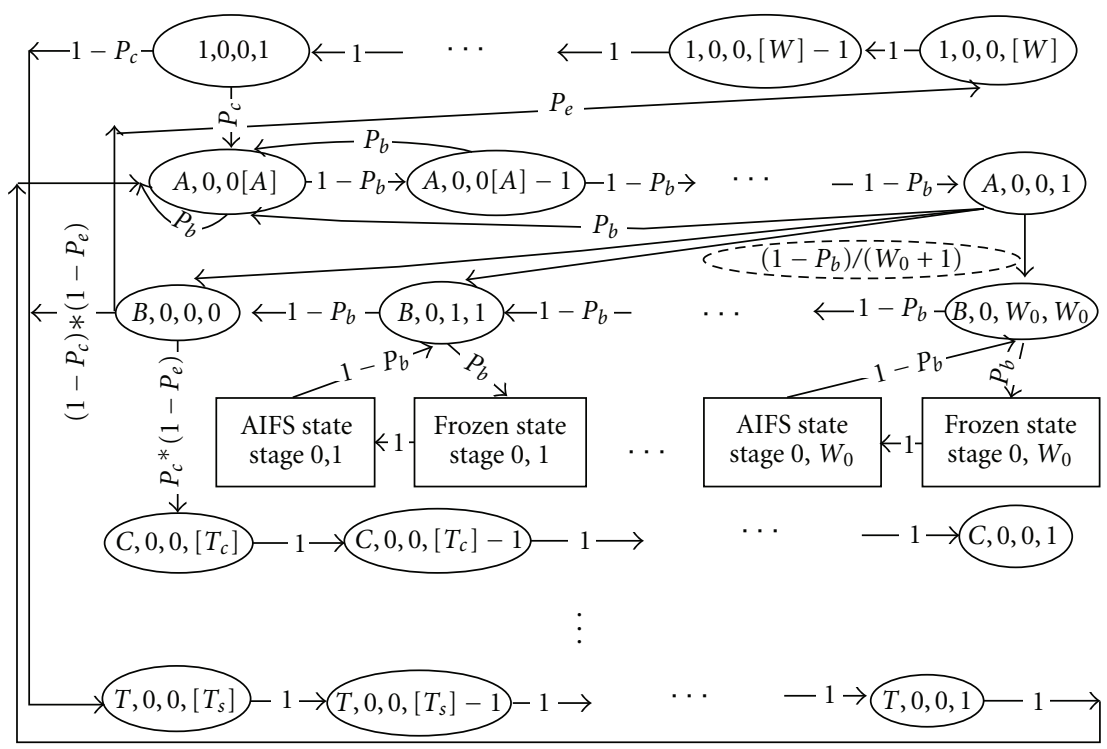

Figure 20: Markov chain extension to general conditions.

$$
P_{B, 0,0,0}=\left[\begin{array}{c}
\frac{\left(1-p_{e}\right) p_{c}\left(1-p_{c}^{m}\right)}{1-p_{c}}\left(1+\left[T_{c}\right]+\frac{1}{p_{b}}\left(\frac{1}{\left(1-p_{b}\right)^{\left[T_{\mathrm{pc}}\right]}}-1\right)\right) \\
+\frac{1}{2\left(1-p_{b}\right)}\left([N] p_{b}+\frac{1}{\left(1-p_{b}\right)^{[A]}}\right)\left(\sum_{j=1}^{m} p_{c}^{j} w_{j}\left(1-p_{e}\right)+w_{0}\right) \\
+1+[W] p_{e}+\frac{1-\left(1-p_{b}\right)^{[A]}}{p_{b}\left(1-p_{b}\right)^{[A]}}+\left[T_{s}\right]\left(1-p_{c}^{m+1}\left(1-p_{e}\right)-p_{c} p_{e}\right)
\end{array}\right]^{-1} .
$$

With these new probabilities, the probability for an $\mathrm{AC}_{i}$ to attempt to access the channel $\tau_{i}$ is

$$
\begin{aligned}
\tau_{i} & =P_{I, 0,0,1}+\sum_{j=0}^{m} P_{B, j, 0,0} \\
& =\frac{1+p_{e i}-p_{c i}^{m+1}\left(1-p_{e i}\right)}{1-p_{c i}} P_{B, 0,0,0}, \quad 0 \leq i \leq 3 .
\end{aligned}
$$

And the probability for the channel to be occupied by the $\mathrm{AC}_{i}$ is

$$
v_{i}=\left(\begin{array}{c}
{\left[T_{s i}\right]\left(1-p_{c i}^{m+1}\left(1-p_{e i}\right)-p_{c i} p_{e i}\right)} \\
+\left[T_{c i}\right]\left(1-p_{e i}\right) \frac{p_{c i}-p_{c i}^{m+1}}{1-p_{c i}} p_{c e x t_{i}}
\end{array}\right) P_{B, 0,0,0,}, \quad 0 \leq i \leq 3 .
$$

Note that if $p_{e}$ is replaced by zero in (37)-(42), which corresponds to the saturation conditions, we get exactly the corresponding equations in the saturation regime.

To resolve the new system equations, two new variables need to be calculated, these are $p_{e i}$ and $\left[W_{i}\right]$. To calculate them, we consider a Poisson arrival process of rate $\lambda$ (packet/s) to model the packets arrival at the MAC buffer of an AC.
For a Poisson process, the probability that the queue is empty after the treatment of the last frame is equal to

$$
p_{e i}=1-\rho_{i}=1-\lambda_{i} D_{i}
$$

where $D_{i}$ is the mean service time, which is here the mean access delay of $\mathrm{AC}_{i}$.

For the average idle time, it is obvious that an AC transits to idle state after the completion of the last transmission and the post-backoff, while the queue remains empty. So, the average waiting time depends on these times as well as on the packet interarrival time using the following equation:

$$
\left[W_{i}\right]=\frac{1}{\lambda_{i}}-D_{i}-\left[T_{s}\right]-T_{P B} .
$$

$1 / \lambda_{i}$ is the packet interarrival time, and $T_{P B}$ is the time needed to achieve the post-backoff procedure.

$$
T_{P B}=D_{A, 0,0,[A]}-D_{B, 0,0,0} \text {. }
$$

7.3. Throughput Analysis. Equation (28) assumes saturation conditions. It is only valid under this assumption and cannot be generalized to all network conditions. To calculate the achievable throughput of each $\mathrm{AC}$ whatever the queue state 
is, our method consists of dividing the network conditions in three different regions and analyzing the achievable throughput in each of these three regions in order to obtain a general solution.

(1) The Saturation Region: $p_{e} \approx 0$. This region is already studied in Section 5, (28) is used here to get the saturated throughput. Let $S_{i \text { sat }}$ be this saturated throughput.

(2) Nonsaturation Region: $p_{e} \approx 1$. Let us see what happens in the interval between two successive transmissions with a non saturated $\mathrm{AC}$ queue. Under the nonsaturation assumption, after each transmission, the AC does a complete post backoff, waits for the average waiting time, and then tries to transmit directly from state $(I, 0,0,1)$. For this attempt, and from this state, the access delay is equal to $p_{c i} * D_{i}+\left(1-p_{c i}\right)$. So, (24) becomes in the nonsaturation case equal to:

$$
S_{\text {insat }}=\frac{M p_{e i} E[P] N_{\text {Transi }}}{T_{P B i}+\left[W_{i}\right]+p_{c i} D_{i}+\left(1-p_{c i}\right)} .
$$

(3) The Saturation Limit Region: $0 \leq p_{e} \leq 1$. To get the throughput in the saturation limit region, we proceed by computing an interpolated value between $S_{i \text { sat }}$ and $S_{\text {insat }}$ with the parameter $p_{e}$. So, the achievable throughput of an $\mathrm{AC}_{i}$ in the saturation limit can be accurately estimated by the following equation:

$$
S_{i}=p_{e i} S_{i n s a t}+\left(1-p_{e i}\right) S_{i s a t} .
$$

Equation (47) gives not only the throughput in the saturation limit, but it is also valid for all network conditions and gives accurate results, as we will see later in the model validation section.

7.4. Delay Analysis. Equations (29) through (35) are applicable to the new Markov chain model, except for $D_{B, 0,0,0}$ because this is the unique state where the transitions are changed as compared to the Markov chain drawn in saturation conditions. From the Markov chain extension in Figure 20, we can see that

$$
\begin{aligned}
D_{B, 0,0,0}= & \left(1-p_{e}\right)\left(1-p_{c}\right)+\left(1-p_{e}\right) p_{c} D_{C, 0,0,[T c]} \\
& +p_{e} p_{c} D_{A, 0,0,[A]}+p_{e}\left(1-p_{c}\right),
\end{aligned}
$$

which gives

$$
D_{B, 0,0,0}=\left(1-p_{c}\right)+p_{c}\left(p_{e} D_{A, 0,0,[A]}+\left(1-p_{e}\right) D_{C, 0,0,[T c]}\right) .
$$
to

After some manipulations, this equation becomes equal

$$
\begin{aligned}
D_{B, 0,0,0}= & \frac{1}{1-p_{c} p_{e}} \\
& \times\left(\begin{array}{c}
\left(1-p_{c}\right)+p_{c}\left(1-p_{e}\right) D_{C, 0,0,\left[T_{c}\right]}+p_{c} p_{e} \\
\times\left(\frac{w_{0}\left(1+[N] p_{b}\left(1-p_{b}\right)^{A}\right)}{2\left(1-p_{b}\right)^{A+1}}+\frac{1-\left(1-p_{b}\right)^{A}}{p_{b}\left(1-p_{b}\right)^{A}}\right)
\end{array}\right),
\end{aligned}
$$

where $D_{C, 0,0,\left[T_{c}\right]}$ is given by

$$
\begin{aligned}
D_{C, 0,0,\left[T_{c}\right]}= & 1+\frac{1+[N] p_{b}\left(1-p_{b}\right)^{A}}{2\left(1-p_{b}\right)^{A+1}} \sum_{i=1}^{m} p_{c}^{i-1} w_{i} \\
& +\frac{1-p_{c}^{m}}{1-p_{c}}\left(\frac{1-\left(1-p_{b}\right)^{T_{\mathrm{pc}}}}{p_{b}\left(1-p_{b}\right)^{T_{\mathrm{pc}}}}+\left[T_{c}\right]\right) .
\end{aligned}
$$

Using (35), we get finally the formulation of $D_{A, 0,0,[A]}$

$$
\begin{aligned}
D_{A, 0,0,[A]}= & D_{B, 0,0,0}+\frac{w_{0}\left(1+[N] p_{b}\left(1-p_{b}\right)^{A}\right)}{2\left(1-p_{b}\right)^{A+1}} \\
& +\frac{1-\left(1-p_{b}\right)^{A}}{p_{b}\left(1-p_{b}\right)^{A}} .
\end{aligned}
$$

From (52), we can easily obtain the formulation of $T_{P B}$ which is defined in (45).

Under general traffic conditions, the starting point of a transmission attempt is not always the state $(A, 0,0,[A])$. Here also, this starting point depends on the state of the transmission queue. Three regions are also to be considered.

(1) The Saturation Region: $p_{e} \approx 0$. In this region, the access delay is equal to $D_{A, 0,0,[A]}$. It can be calculated by the use of (36) or (52). These two equations are equivalent when $p_{e}$ is replaced by zero in the latter one.

(2) Nonsaturation Region: $p_{e} \approx 1$. In this region, the station accomplishes a complete post-backoff and a complete idle period before the arrival of a new packet. The starting point of the transmission attempt is here the state $(I, 0,0$, and 1$)$ and the access delay is equal to $D_{I, 0,0,1}$

$$
D_{I, 0,0,1}=p_{c} D_{A, 0,0,[A]}+\left(1-p_{c}\right) .
$$

(3) The Saturation Limit Region: $0 \leq p_{e} \leq 1$. In this region, which reflects the general case, we adopt the same reasoning used in throughput calculation. So, the following equation computes the access delay:

$$
\begin{aligned}
D_{i} & =p_{e i} D_{I, 0,0,1}+\left(1-p_{e i}\right) D_{A, 0,0,[A]} \\
& =\left(p_{e i} p_{c i}+1-p_{e i}\right) D_{A, 0,0,[A]}+p_{e i}\left(1-p_{c i}\right) .
\end{aligned}
$$

Equation (54) is used to get the mean access delay for all network conditions.

Note finally that as in saturation conditions, the development of our equations gives a unique mathematical equation for the throughput computation and another unique one for the delay computation.

\section{Model Validation}

To validate our complete model, the queues must pass from extremely nonsaturated to extremely saturated conditions. In order to obtain such behavior, we choose a fixed number of wireless stations and we change the packet arrival rate. 


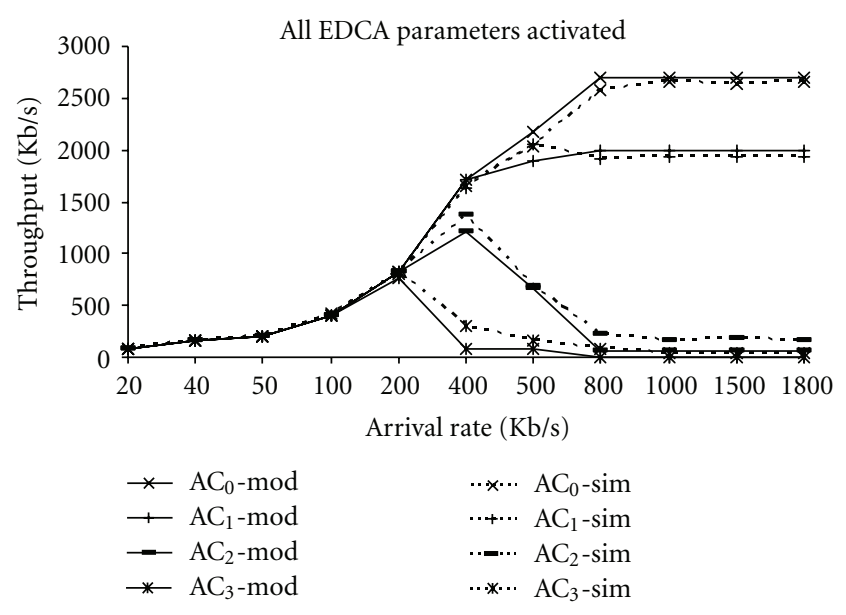

Figure 21: Achievable throughput per AC-all parameters activated.

Therefore, the network topology described in Section 4.2, and drawn in Figure 2 is very convenient to this set of simulations.

Our goal here is to prove the accuracy of the proposed model concerning the modeling of the three differentiation parameters: AIFS, CW and TXOPLimit. The objective here is not to evaluate the effect of each of these parameters but rather to verify that their effects are well modeled and reproduced by our analytical model. The parameters values for these simulations are summarized in Table 4.

In Figures 21-32, we traced the results we obtained from these simulations as well as from the numerical calculations.

To be sure of the accuracy of the simulation values, and to be aware of the effect of simulation time, each simulation was done nine times, and each time we change the simulation time by adding one additional minute. The simulation times go from $60 \mathrm{~s}$ to $540 \mathrm{~s}$, after a stabilization time equal to $60 \mathrm{~s}$. Therefore, each simulation value drawn in these figures is the average of nine different values resulting from nine simulation runs with the same conditions but with different simulation times. This technique is already used in the literature in order to limit the simulation errors [12]. Note also that this set of simulations has also been performed with different starting times of different AC's traffic. The results obtained were similar to those presented here.

In simulation 1, EDCA default parameters are used. All the differentiation parameters are active. Figures 21, 22, and 23 show the obtained results. It is very clear in these figures that the model's results match the simulation results regarding the three differentiation parameters modeling. These default parameters are the standard recommended ones, and they are suitable for the case where all the four ACs are active at the same time. We can clearly see in Figure 21 how $\mathrm{AC}_{1}$ (video) gains more throughputs in the saturation zone in favor of $\mathrm{AC}_{2}(\mathrm{BE})$. We can also see in Figure 23 how the delays of $\mathrm{AC}_{0}$ (voice) and $\mathrm{AC}_{1}$ (video) remain relatively low as compared to $\mathrm{AC}_{2}$ and $\mathrm{AC}_{3}$ satisfying their applications constraints. The total achievable throughput is approximately equal to $4.9 \mathrm{Mb} / \mathrm{s}$ in the saturation zone (Figure 22).

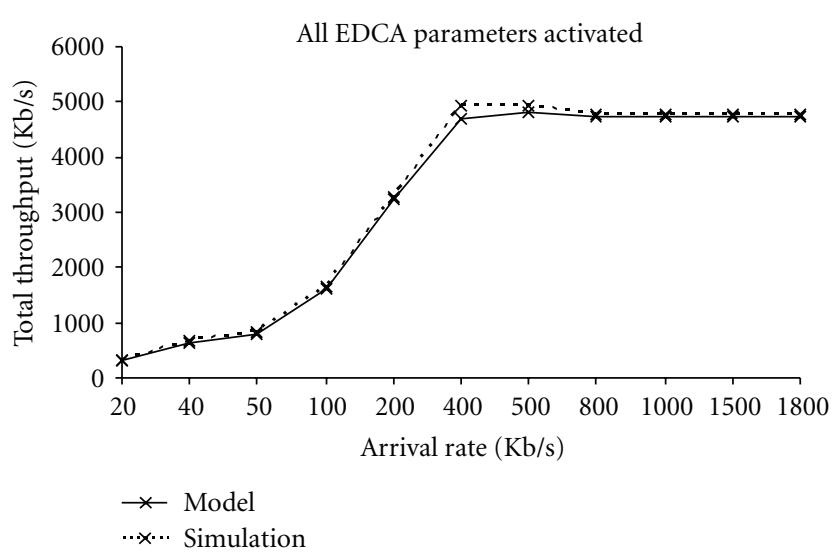

FIGURE 22: Total achievable throughput—all parameters activated.

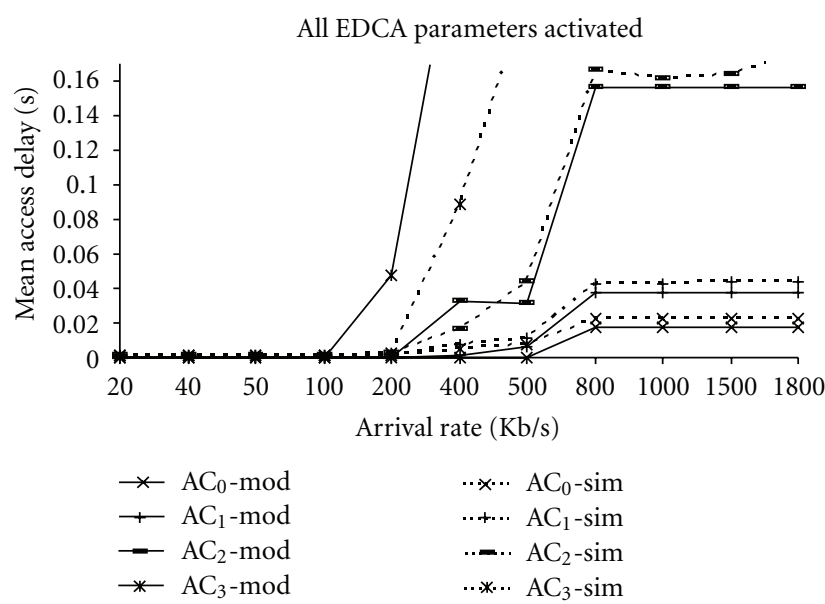

Figure 23: Access delay per AC—all parameters activated.

In simulation 2, the TXOPLimit differentiation parameter is deactivated. Figures 24, 25, and 26 and show the corresponding results. The accuracy of the model in predicting the performance metrics is also verified in this case. Compared to the simulation 1, this simulation case shows the effect of deactivating TXOPLimit on the global performance. We can see in Figure 24 how the saturated throughput of $\mathrm{AC}_{1}$ cannot reach the same value obtained in Figure 21 and how $\mathrm{AC}_{2}$ can gain more throughput as compared to the default values case. Because of this configuration, the total achievable throughput in the saturation zone cannot exceed the value of $4 \mathrm{Mb} / \mathrm{s}$, which is less than the maximum achievable throughput realized in simulation $1(4.9 \mathrm{Mb} / \mathrm{s})$.

From simulations 1 and 2, one can note that our analytical model allows us to reproduce finely the effect of the TXOPLimit on the global performance.

In simulation 3, the AIFS differentiation parameter is deactivated. Figures 27, 28, and 29 show the results for this case. The matching between analytical curves and simulation again proves that our analytical model gives a very good estimation of the performance metrics even if we deactivate the AIFS differentiation parameter. In this case, AIFS is set to 2 for all ACs. Therefore, $\mathrm{AC}_{2}$ and $\mathrm{AC}_{3}$ will have the same EDCA parameter values. It is thus obvious in this case that 
TABLE 4: EDCA parameters for different simulation scenarios.

\begin{tabular}{lccrr}
\hline & AIFSN & $\mathrm{CW}_{\min }$ & $\mathrm{CW}_{\max }$ & TXOPLimit \\
\hline Sim. 1 & $\left(\mathrm{AC}_{0}, \mathrm{AC}_{1}, \mathrm{AC}_{2}, \mathrm{AC}_{3}\right)$ & & $(3264,6016,0,0)$ \\
Sim. 2 & $(2,2,3,7)$ & $(7,15,31,31)$ & $(15,31,1023,1023)$ & $(0,0,0,0)$ \\
Sim. 3 & $(2,2,3,7)$ & $(7,15,31,31)$ & $(15,31,1023,1023)$ & $(3264,6016,0,0)$ \\
Sim. 4 & $(2,2,2,2)$ & $(7,15,31,31)$ & $(15,31,1023,1023)$ & $(3264,6016,0,0)$ \\
\hline
\end{tabular}

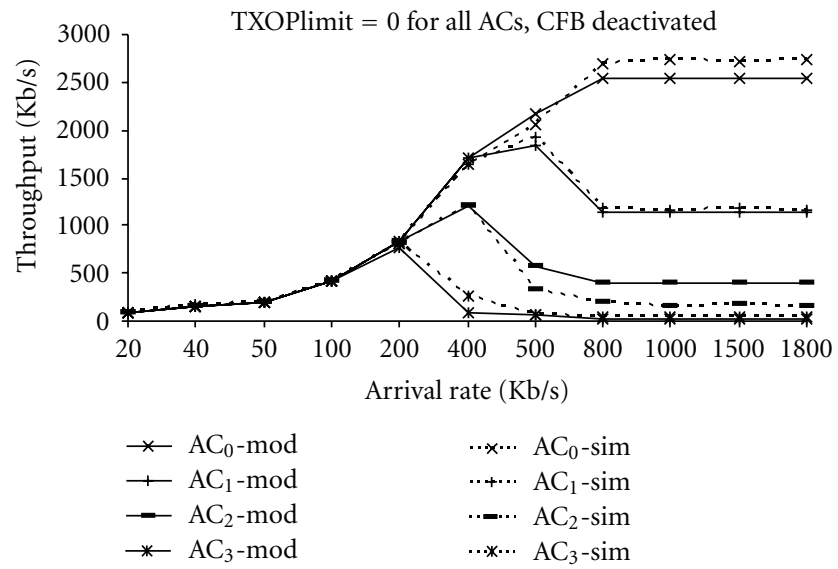

Figure 24: Achievable throughput per AC-TXOPLimit deactivated.

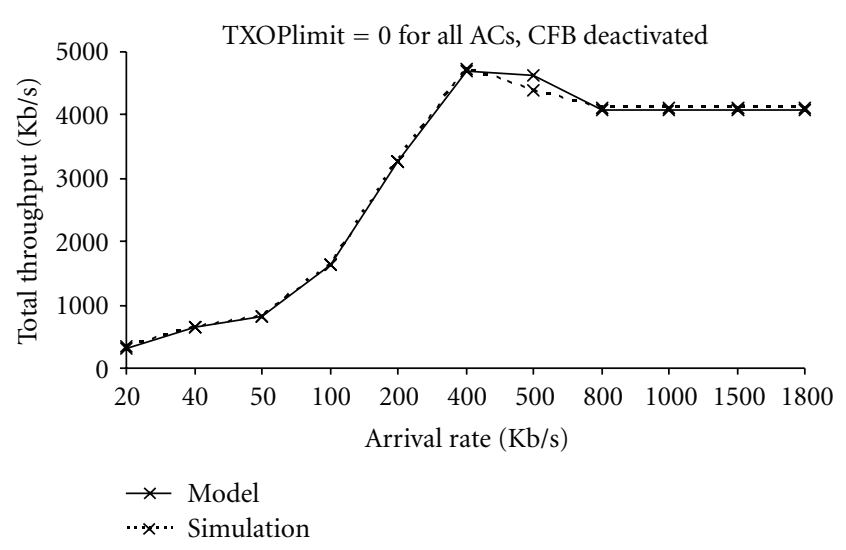

FIgUre 25: Total achievable throughput-TXOPlimit deactivated.

we get the same achievable throughput and access delay for these two ACs and this is what is obtained by our model as noticed in Figures 27 and 29. Here, also the effect of the AIFS differentiation parameters is well reported by our analytical model.

In simulation 4 , the $\mathrm{CW}$ differentiation parameter is deactivated. Figures 30, 31, and 32 show the results obtained. In this last case, the accuracy of the model is also demonstrated. In this case, $\mathrm{CW}_{\min }$ is set to 7 and $\mathrm{CW}_{\max }$ is set to 15 for all ACs. So, $\mathrm{AC}_{1}$ becomes more prioritized than $\mathrm{AC}_{0}$. In fact, with this configuration, $\mathrm{AC}_{1}$ is privileged by the TXOPLimit parameter. For this reason, it gets more throughput than $\mathrm{AC}_{0}$, and approximately the same access delay as $\mathrm{AC}_{0}$. Having the same AIFS and the same $\mathrm{CW}_{\text {min }}$

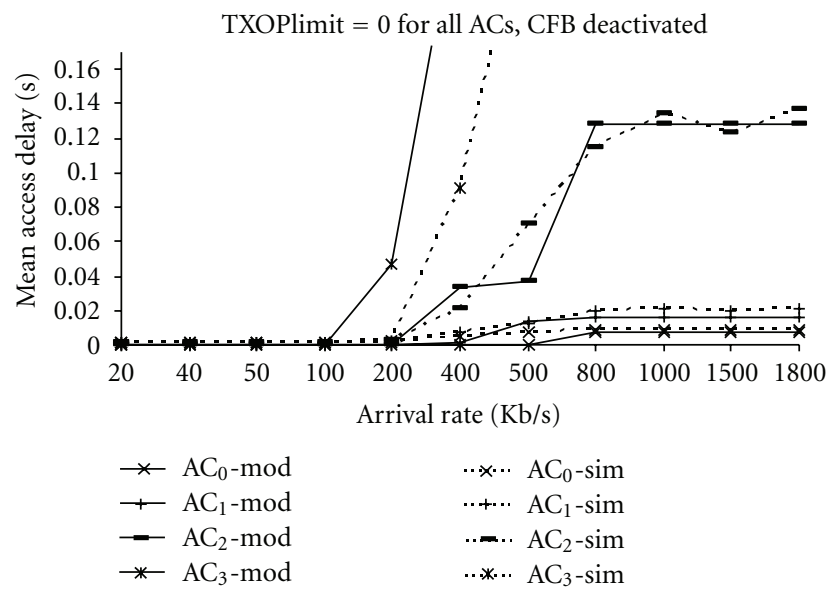

Figure 26: Access delay per AC-TXOPLimit deactivated.

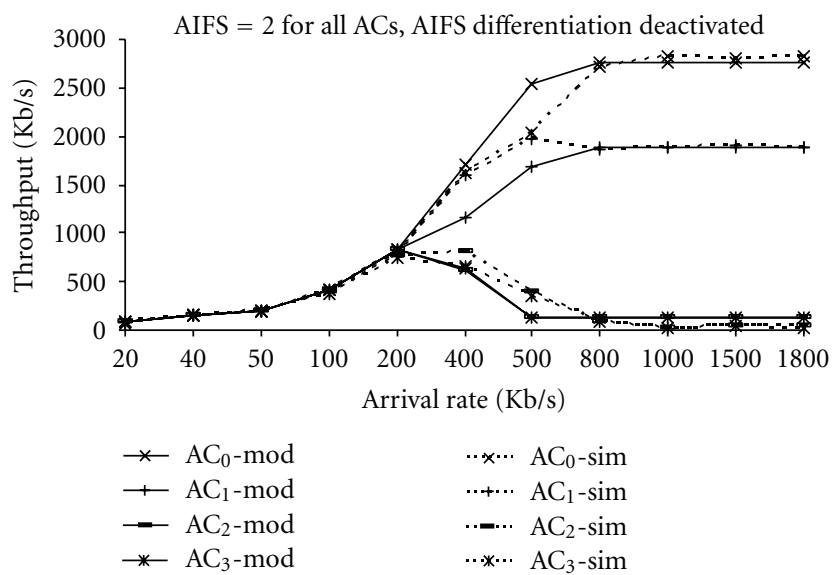

FIgURE 27: Achievable throughput per AC-AIFS deactivated.

and $\mathrm{CW}_{\max }, \mathrm{AC}_{0}$ and $\mathrm{AC}_{1}$ get the same access delays. This can be shown in Figures 30 and 32. This is also verified for $\mathrm{AC}_{2}$ when we compare between its access delay in Figure 32 and that in Figure 23. We see that lower values for $\mathrm{CW}_{\text {min }}$ and $\mathrm{CW}_{\max }$ lead to lower values for the access delay. This is noticed in both the analytical and the simulation results.

To sum up, the nearly-exact match between the analytical and the simulation curves shown in Figure 21 through Figure 32 proves that our model gives a very good estimation of the performance metrics for any of the configurations of the differentiation parameters. Therefore, globally, the analytical model proposed for all network conditions gives very satisfactory results. It models accurately the three differentiation parameters and provides a very good prediction. 


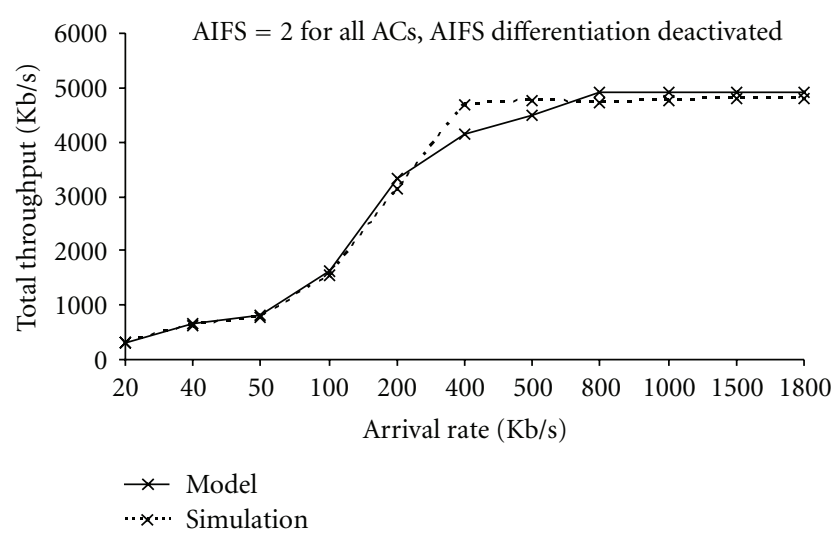

FIgUre 28: Total achievable throughput —AIFS deactivated.

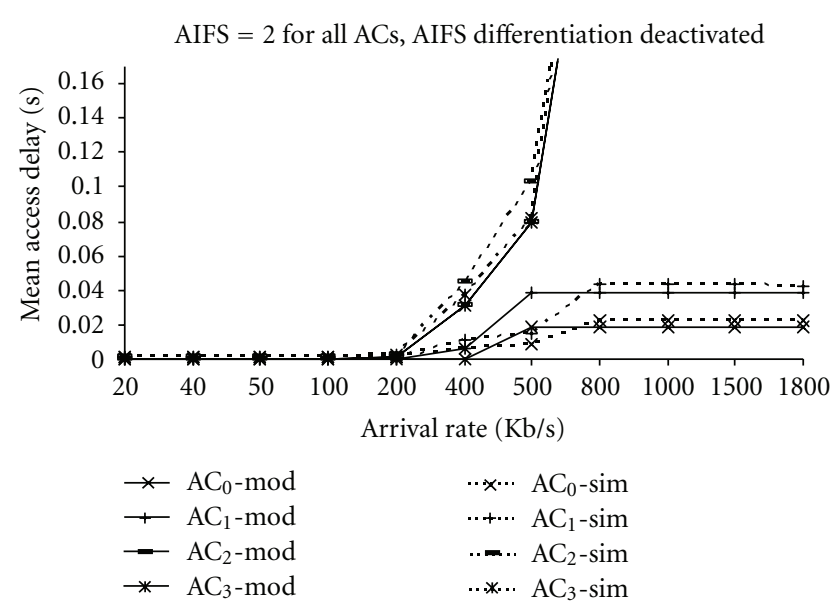

Figure 29: Access delay per AC-AIFS deactivated.

If we go further into details in these curves and if we distinguish between the three regions, nonsaturation, within the saturation limit, and in saturation conditions, we can extract the following interpretations.

(a) In the nonsaturation region $(<200 \mathrm{~Kb} / \mathrm{s}$ per $\mathrm{AC}$ per station), all the packets are served by the network with very low access delays. All ACs get the necessary bandwidth to serve the incoming packets. The achievable throughput for each $\mathrm{AC}$ is equal to its arrival rate. In this region, the model is extremely accurate in predicting both the throughput and the access delay. We can clearly note a negligible difference between simulation and analytical results, which is less than $0.5 \%$ for all ACs.

(b) In the saturation region $(>800 \mathrm{~Kb} / \mathrm{s}$ per $\mathrm{AC}$ per station), the achievable throughput and the access delay reach the achievable values in saturation and remain constant. The total achievable throughput is distributed among the ACs according to their priorities. This distribution depends on the EDCA parameters values of these ACs. The model provides also a very good accuracy in this region. The difference between simulation and analytical results does not exceed 3\% for all ACs.

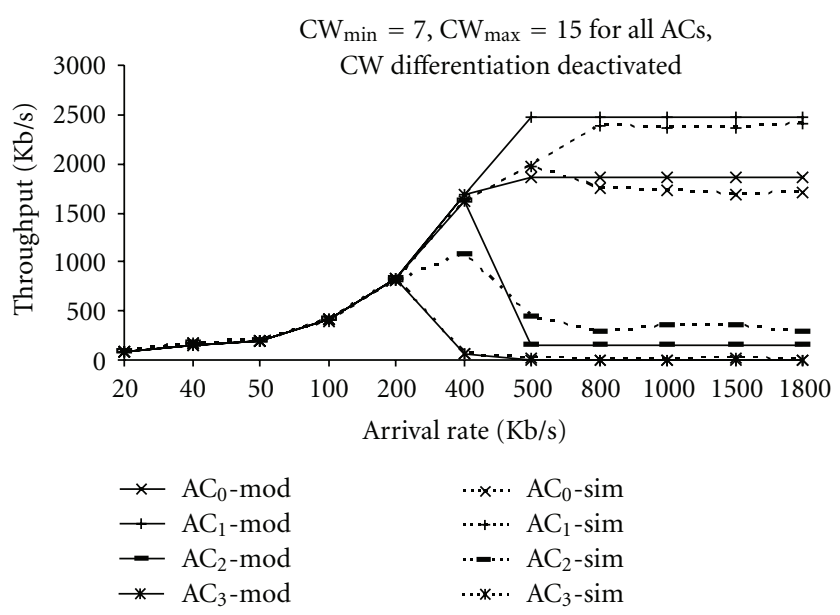

FIgURE 30: Achievable throughput per AC_-CW deactivated.

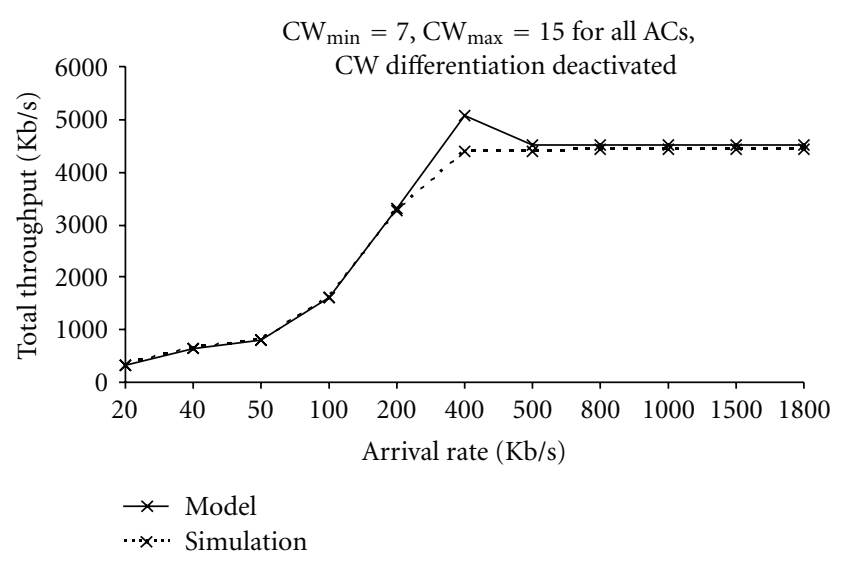

FIGURE 31: Total achievable throughput-CW deactivated.

(c) Within the saturation limit region (between $200 \mathrm{~Kb} / \mathrm{s}$ and $800 \mathrm{~Kb} / \mathrm{s}$ per $\mathrm{AC}$ per station), we notice an estimation error between the analytical model results and the simulation results. This error is approximately equal to $10.83 \%$ for $\mathrm{AC}_{0}, 10.66 \%$ for $\mathrm{AC}_{1}$, $10.84 \%$ for $\mathrm{AC}_{2}$ and finally $10.09 \%$ for $\mathrm{AC}_{3}$. This is due to two effects. First, it is in this region that the effect of the queue model assumption is introduced. This effect is already analyzed in [32], and we proved also that this error is mitigated by the global performance of the model and does not affect seriously the global accuracy of the model. Second, our approach of using an interpolated value between the saturation and nonsaturation values based on the value of $p_{e}$ can have an impact on this fluctuation error. This error, being low and not exceeding $11 \%$ on average, does not affect the global accuracy of the model.

Some other common and general conclusions about the behavior of the EDCA MAC protocol can also be drawn from the results traced in Figures 21 and 32.

(1) In the saturation region, the throughput and the delay remain constant. Hence, for a fixed number 
of active stations, the packet arrival rate does not affect the achievable throughput and delay. But as demonstrated in Section 6, the addition of a new active station affects the achievable throughput and delay.

(2) The saturated throughput is not the maximum achievable throughput. Some ACs (especially $\mathrm{AC}_{0}$ and $\mathrm{AC}_{1}$ ) get more throughput within the saturation limit region than in the saturation region. This result outlined by our analytical model was also demonstrated in some simulation studies as well as by older models [10-13]. The maximum protocol capacity can only be achieved in the nonsaturated case. This proves another time the need for a complete analytical model to make an efficient admission control that should maintain the system in the nonsaturated region to maximize the capacity usage.

(3) Finally, the performance metrics for each access category are highly influenced by the choice of the EDCA differentiation parameters. This can be shown if we compare the values obtained from the four above simulations. The choice of AIFS and CW has a great impact on the access delay, and the choice of TXOPLimit has a great impact on the achievable throughput. A correct parameter's setting is a very important task when all of the ACs exist together in the network, in order to privilege some traffic over the others.

We can conclude this section by saying that our proposed analytical model offers a very good numerical tool to: accurately estimate the throughput and the access delay in general network conditions and for any of the configurations of the differentiation parameters. The model covers all the features of the EDCA procedure and consequently can be considered as the one that covers all the drawbacks in other existing models in the literature. Thus, it will constitute the best numerical tool to date for an efficient admission control algorithm.

\section{Computational Aspects}

Analytical models for 802.11e EDCA must be implemented in an efficient admission control algorithm. Hence, it is highly recommended that such an algorithm gets a low computational overhead. Obviously, the use of nonlinear equations model lead to higher computational complexity when compared to models based on mean value analysis. But the low computational overhead is not the only criteria to be examined in an analytical model. Having a low computational cost model, with estimated, non accurate QoS metrics, is not desired at all. Indeed, such model leads to a non efficient admission control algorithm. Therefore, the used model must provide a compromise between good accuracy and low complexity.

The model we proposed in this paper is a non linear computational one (four dimensional Markov chain). The first dimension in our Markov chain is used to distinguish

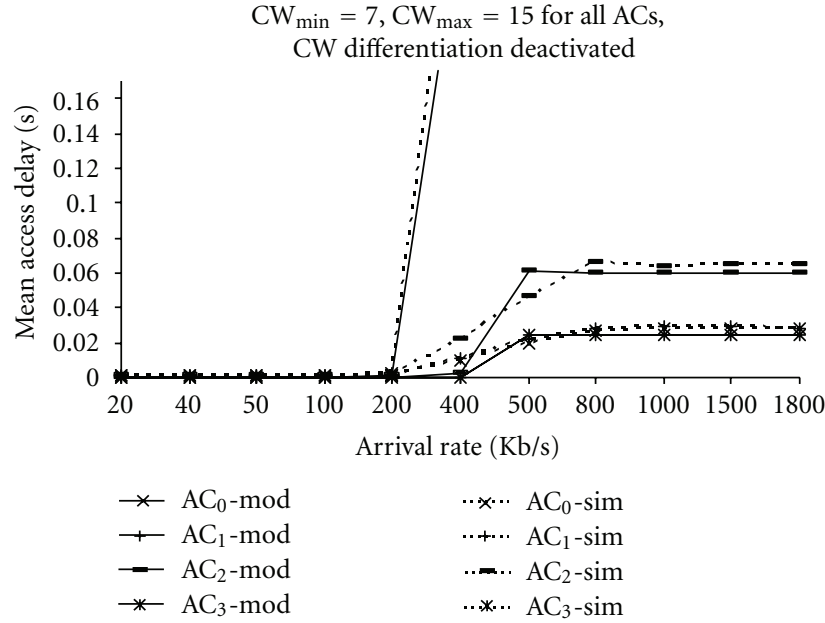

Figure 32: Access delay per AC—CW deactivated.

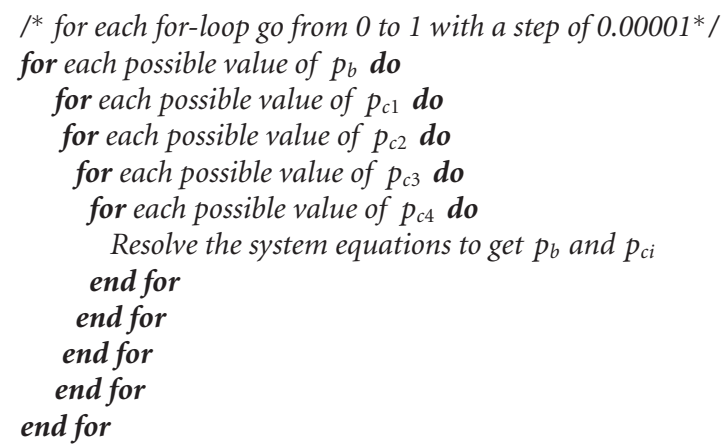

Calculate throughput and delay using $p_{b}$ and $p_{c i}$

Algorithm 1: Full search for the solution.

between different states allowing an understandable way to model EDCA features. So, it implies no computational overhead and it allows us to simplify the complexity of the model. Only the remaining three dimensions are used in the calculations. Let us also remind that in Section 3, we have demonstrated the need for a three dimensional Markov chain to accurately implement the differentiation features in EDCA. So, a three dimensional model is the minimum possible needed to obtain good and accurate results.

Different features of our model help to reduce the computational overhead and the execution time needed to calculate the throughput and the access delay for each AC. Indeed, in our model, we try to obtain the close-form expressions which simplify the computational costs. Hence, in the throughput and delay calculation, the single given expressions reduce completely the computational overhead related to the calculation of these metrics, and once the system is resolved, the throughput and the access delay are calculated immediately.

Also in the non linear system resolution, we have 5 nested "for loops", one for $p_{b}$ and four for $p_{c}$ (i.e., one for each AC). Of course if the program tries all possible values for $p_{b}$ and $p_{c i}$, it will lead to a high complexity. This is illustrated in 


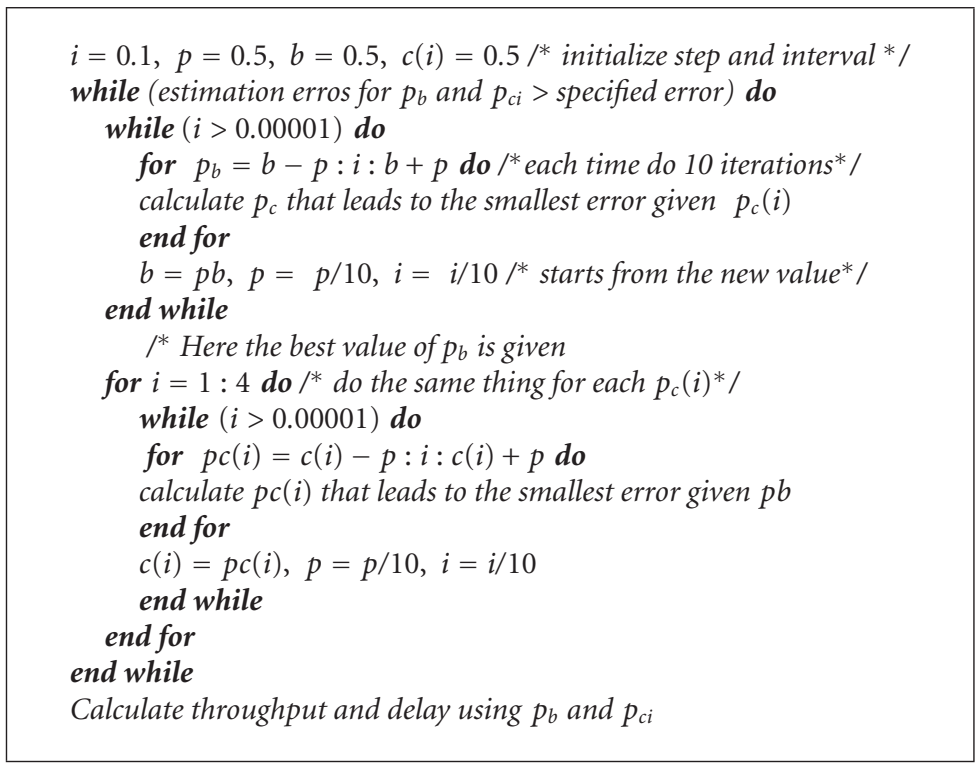

Algorithm 2: Optimized search for the solution.

Algorithm 1, where we need to perform a very high number of iterations to get the solution. Therefore, we suggested here the use of a simple method consisting of searching the value that leads to the smallest estimation error in a given interval, and then we recalculate around this value till we get the solution as illustrated in Algorithm 2. This method greatly reduces the calculation time and gets the exact solution with a very small estimation error $\left(\sim 10^{-6}\right)$ in a short period of time. For instance, this is performed in approximately $4 \mathrm{~s}$ (using Matlab 7, on a Pentium IV $2.4 \mathrm{GHz}$ personal computer with 512 Mo RAM). Another aspect to be considered here is the delay and the $p_{e}$ calculation within the system resolution. In fact, the access delay depends on $p_{e}$ and $p_{e}$ depends on the access delay. A simple "while loop" is used to obtain the convergence of these two values with a very low computational time. So, our model, in addition to some basic algorithmic simplifications, is able to surpass the complexity of our four-dimensional Markov chain and gives accurate results for admission control purposes in a very satisfactory calculation time.

\section{Conclusion}

In this paper, we have presented a four-dimensional Markov chain model for EDCA, as described in the final standard [1]. Our model allows us to compute the available throughput and mean access delay within each $\mathrm{AC}$ whatever are the traffic conditions, going from extremely nonsaturated to extremely saturated network. The model proposed is the only one in the literature that models all the features of EDCA.

After having highlighted the influence of the CFB mode on 802.11e performance and the importance of taking it into account when modeling the 802.11e EDCA function, we have proceeded into the presentation of a simple numerical equation to calculate the transmission time in CFB mode, where an AC can transmit a burst of frames when accessing the channel using the TXOPLimit differentiation parameter.
As far as we know, this is the first time this feature is modeled. We have demonstrated by means of simulations that the simple CFB model we have proposed is accurate indeed.

We have then explained our approach in modeling 802.11e EDCA in order to calculate the performance metrics such as throughput and delay. The model is first drawn in saturation conditions. Comparison with both a reference model and simulation results shows that the model is extremely accurate in predicting the per-AC saturated throughput and access delay.

Finally, we have extended the model to cover all network conditions, and we have presented our complete analytical model for EDCA. Its validation showed that this latest one provides us with a powerful tool to develop a call admission control scheme for further QoS improvements in WLANs. In fact, this model was developed with two main objectives: to have the most accurate prediction of throughput and access delay and to have a low computational complexity. These are the two main requirements for an efficient admission control algorithm. This admission control algorithm constitutes the next step in our research.

\section{References}

[1] IEEE P802.11e/D13.0, "Wireless medium access control (MAC) and physical layer (PHY) specifications," Medium Access Control (MAC) Quality of Service (QoS) Enhancements, 2005.

[2] G. Bianchi, "Performance analysis of the IEEE 802.11 distributed coordination function," IEEE Journal on Selected Areas in Communications, vol. 18, no. 3, pp. 535-547, 2000.

[3] H. Wu, Y. Peng, K. Long, S. Cheng, and J. Ma, "Performance of reliable transport protocol over IEEE 802.11 wireless LAN: analysis and enhancement," in Proceedings of the IEEE Infocom, pp. 599-607, New York, NY, USA, June 2002.

[4] K. Xu, Q. Wang, and H. Hassanein, "Performance analysis of differentiated QoS supported by IEEE 802.11e enhanced distributed coordination function (EDCF) in WLAN," in 
Proceedings of the IEEE Global Telecommunications Conference (GLOBECOM '03), pp. 1048-1053, San Francisco, Calif, USA, December 2003.

[5] Y. Xiao, "Performance analysis of IEEE 802.11e EDCF under saturation condition," in Proceedings of the IEEE International Conference on Communications (ICC '04), pp. 170-174, Chicago, Ill, USA, June 2004.

[6] J. Hui and M. Devetsikiotis, "A unified model for the performance analysis of IEEE 802.11e EDCA," IEEE Transactions on Communications, vol. 53, no. 9, pp. 1498-1510, 2005.

[7] Z. N. Kong, D. H. K. Tsang, B. Bensaou, and D. Gao, "Performance analysis of IEEE 802.11e contention-based channel access," IEEE Journal on Selected Areas in Communications, vol. 22, no. 10, pp. 2095-2106, 2004.

[8] H. Wu, X. Wang, Q. Zhang, and X. Shen, "IEEE 802.11e Enhanced Distributed Channel Access (EDCA) throughput analysis," in Proceedings of the IEEE International Conference on Communications (ICC '06), pp. 223-228, Montreal, Canada, July 2006.

[9] P. E. Engelstad and O. N. Østerbø, "Non-saturation and saturation analysis of IEEE 802.11e EDCA with starvation prediction," in Proceedings of the 8th ACM Symposium on Modeling, Analysis and Simulation of Wireless and Mobile Systems, pp. 224-233, Montreal, Canada, October 2005.

[10] D. Malone, K. Duffy, and D. Leith, "Modeling the 802.11 distributed coordination function in nonsaturated heterogeneous conditions," IEEE/ACM Transactions on Networking, vol. 15, no. 1, pp. 159-172, 2007.

[11] F. Y. Hung and I. Marsic, "Analysis of non-saturation and saturation performance of IEEE 802.11 DCF in the presence of hidden stations," in Proceedings of the IEEE 66th Vehicular Technology Conference (VTC '07), pp. 230-234, Baltimore, Md, USA, October 2007.

[12] P. E. Engelstad and O. N. Østerbø, "Analysis of the total delay of IEEE 802.11e EDCA and 802.11 DCF," in Proceedings of the IEEE International Conference on Communications (ICC '06), pp. 552-559, Istanbul, Turkey, July 2006.

[13] W. Zhang, J. Sun, J. Liu, and H. B. Zhang, "Performance analysis of IEEE 802.11e EDCA in wireless LANs," Journal of Zhejiang University, vol. 8, no. 1, pp. 18-23, 2007.

[14] E. Ziouva and T. Antonakopoulos, "CSMA/CA performance under high traffic conditions: throughput and delay analysis," Computer Communications, vol. 25, no. 3, pp. 313-321, 2002.

[15] M. Ergen and P. Varaiya, "Throughput analysis and admission control for IEEE 802.11a," Mobile Networks and Applications, vol. 10, no. 5, pp. 705-716, 2005.

[16] J. S. Vardakas, I. Papapanagiotou, M. D. Logothetis, and S. A. Kotsopoulos, "On the end-to-end delay analysis of the IEEE 802.11 distributed coordination function," in Proceedings of the 2nd International Conference on Internet Monitoring and Protection (ICIMP '07), Silicon Valley, Calif, USA, July 2007.

[17] A. Abdrabou and W. Zhuang, "Service time approximation in IEEE 802.11 single-hop ad hoc networks," IEEE Transactions on Wireless Communications, vol. 7, no. 1, pp. 305-313, 2008.

[18] Y. C. Tay and K. C. Chua, "A capacity analysis for the IEEE 802.11 MAC protocol," Wireless Networks, vol. 7, no. 2, pp. 159-171, 2001.

[19] "Wireless medium access control (MAC) and physical layer (PHY) specifications: medium access control (MAC) Quality of Service (QoS) Enhancements," IEEE P802.11e/D13.0, 2005.

[20] J. W. Robinson and T. S. Randhawa, "Saturation throughput analysis of IEEE 802.11e enhanced distributed coordination function," IEEE Journal on Selected Areas in Communications, vol. 22, no. 5, pp. 917-928, 2004.
[21] Y. Xiao, "Performance analysis of priority schemes for IEEE 802.11 and IEEE 802.1 le wireless LANs," IEEE Transactions on Wireless Communications, vol. 4, no. 4, pp. 1506-1515, 2005.

[22] T. C. Tsai and M. J. Wu, "An analytical model for IEEE 802.11e EDCA," in Proceedings of the IEEE International Conference on Communications (ICC '05), pp. 3474-3478, Seoul, Korea, May 2005.

[23] J. W. Tantra, C. H. Foh, and A. B. Mnaouer, "Throughput and delay analysis of the IEEE 802.11e EDCA saturation," in Proceedings of the IEEE International Conference on Communications (ICC'05), pp. 3450-3454, Seoul, Korea, May 2005.

[24] Z. Tao and S. Panwar, "Throughput and delay analysis for the IEEE 802.11e enhanced distributed channel access," IEEE Transactions on Communications, vol. 54, no. 4, pp. 596-603, 2006.

[25] Y. Lin and V. W. S. Wong, "Saturation throughput of IEEE 802.11e EDCA based on mean value analysis," in Proceedings of the IEEE Wireless Communications and Networking Conference (WCNC '06), pp. 475-480, Las Vegas, Nev, USA, April 2006.

[26] L. Xiong and G. Mao, "Saturated throughput analysis of IEEE 802.11e EDCA," Computer Networks, vol. 51, no. 11, pp. 30473068, 2007.

[27] Y. Yan and C. Pan, "An improved analytical model for IEEE802.11e enhanced distributed channel access," in Proceedings of the International Symposium on Information Technology Convergence (ISITC '07), pp. 135-139, Jeonju, Korea, November 2007.

[28] P. E. Engelstad and O. N. Østerbø, "Delay and throughput analysis of IEEE 802.11e EDCA with starvation prediction," in Proceedings of the IEEE Conference on Local Computer Networks (LCN '05), pp. 647-654, Sydney, Australia, November 2005.

[29] P. E. Engelstad and O. N. Østerbø, "Analysis of the total delay of IEEE 802.11e EDCA and 802.11 DCF," in Proceedings of the IEEE International Conference on Communications (ICC '06), pp. 552-559, Montreal, Canada, July 2006.

[30] I. Inan, F. Keceli, and E. Ayanoglu, "Modeling the 802.11e enhanced distributed channel access function," in Proceedings of the 50th Annual IEEE Global Telecommunications Conference (GLOBECOM '07), pp. 2546-2551, Washington, DC, USA, November 2007.

[31] X. Ling, K. H. Liu, Y. Cheng, X. Shen, and J. W. Mark, "A novel performance model for distributed prioritized MAC protocols," in Proceedings of the 50th Annual IEEE Global Telecommunications Conference (GLOBECOM '07), pp. 46924696, Washington, DC, USA, November 2007.

[32] N. Chendeb, Y. Ghamri-Doudane, and B. El Hassan, "Effect of transmission opportunity limit on transmission time modeling in 802.11e," in Proceedings of the 7th IEEE International Workshop on IP Operations and Management (IPOM '07), pp. 156-167, San José, Calif, USA, 2007.

[33] S. Mangold, S. Choi, G. R. Hiertz, O. Klein, and B. Walke, "Analysis of IEEE 802.11e For QOS Support In Wireless LANS," Wireless Communications, vol. 10, no. 6, pp. 40-50, 2003.

[34] The Network Simulation-ns-2, http://www.isi.edu/nsnam/ ns/.

[35] IEEE 802.11e implementation for ns-2, http://www.tkn.tuberlin.de/research/802.11e_ns2/. 

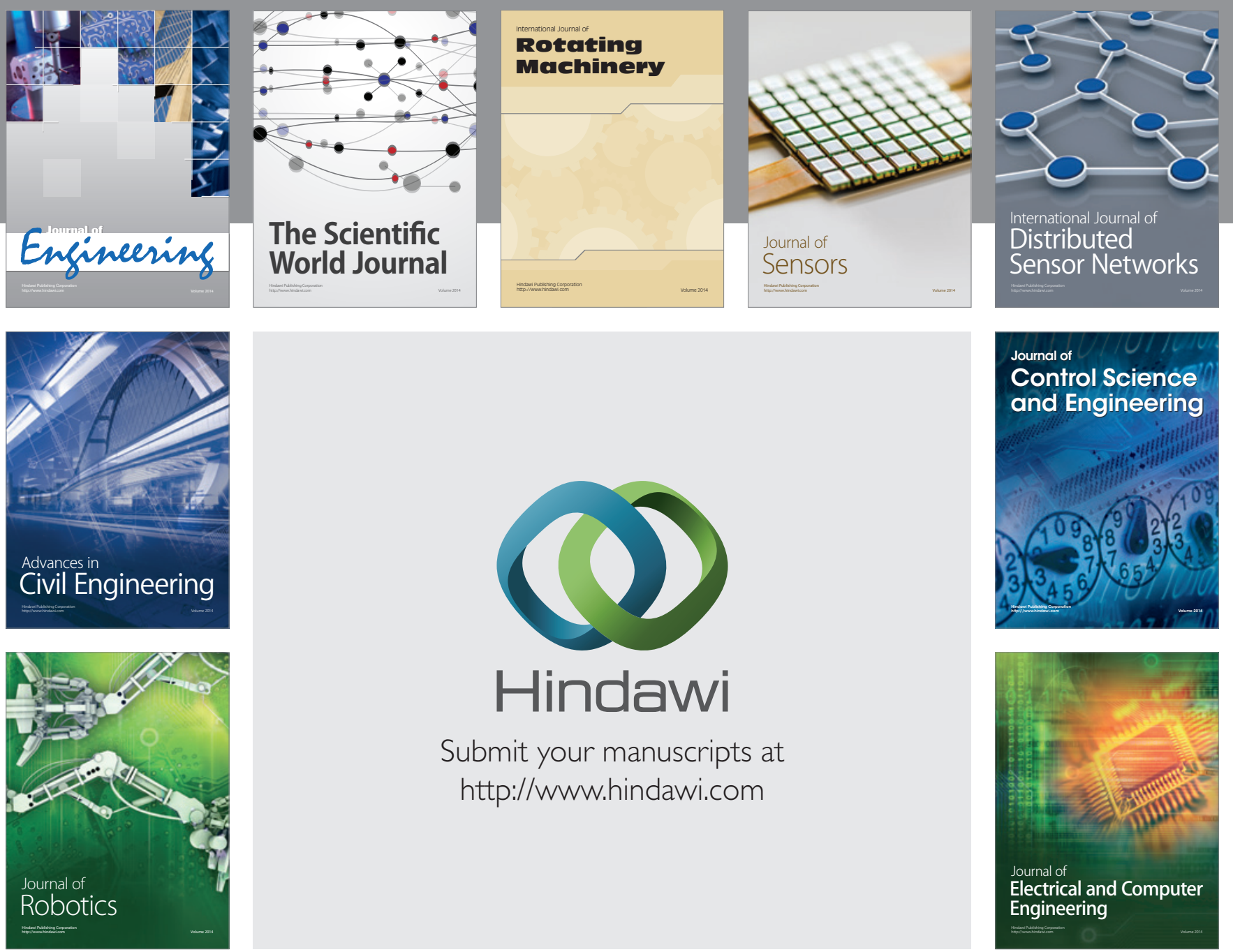

Submit your manuscripts at

http://www.hindawi.com
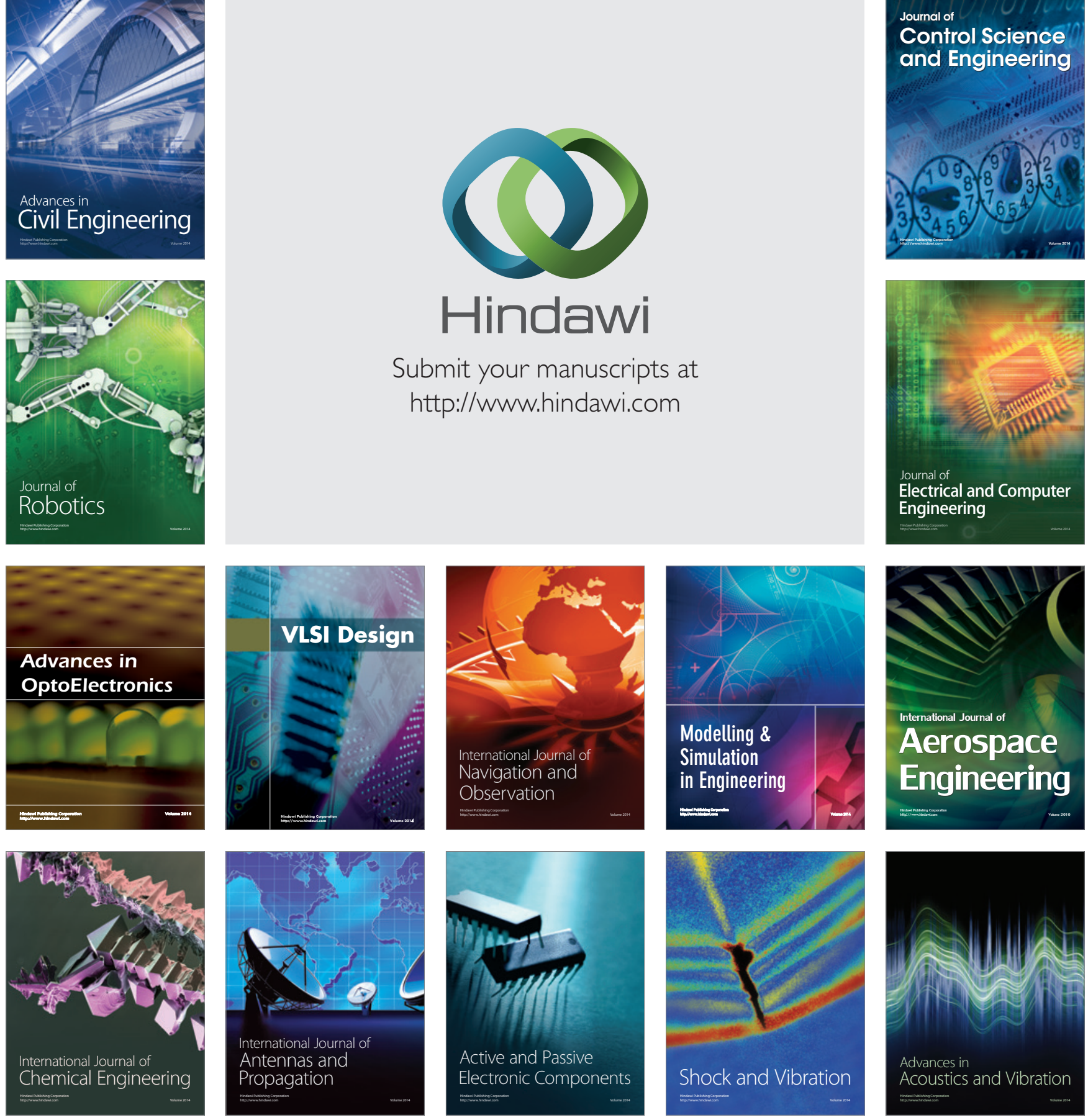